Final Report Certification
for
CRADA Number _ ORNL o1-0624

Between

UT-Battelle, LLC

and

IDA Research

(Participant)

Instructions:

Mark the appropriate statement in 1a or $1 \mathrm{~b}$ below with an IX" Refer to the articles in the CRADA terms and conditions governing the identification and marking of Protected CRADA Information (PCl).

If no PCl is identified, the report will be distributed without restriction. If $\mathrm{PCl}$ is identified, the report distribution will be limited in accordance with the CRADA terms and conditions governing release of data. In all cases items 2 and 3 must be true That is, the report cannot contain Proprietary Information and a disclosure must be filed prior to release of the report

This certification may either be made by using this form or may be made on company letterhead if the Participant desires. A faxed copy of this completed form is acceptable.

The following certification is made for the subject final report:

1. (a) The final report contains information that qualifies as "Protected CRADA Information" (PCl).. The PCl legend is printed on the report cover, and the $\mathrm{PCl}$ is clearly identified.

OR

(6) The final report does not contain "Protected CRADA Information." The "Approved for Public Release" legend is printed on the report cover.

2. The final report does not contain Proprietary information.

3. By the signature below, the Participant has no objection to the public distribution of the final report due to patentable information.

For the Participant:
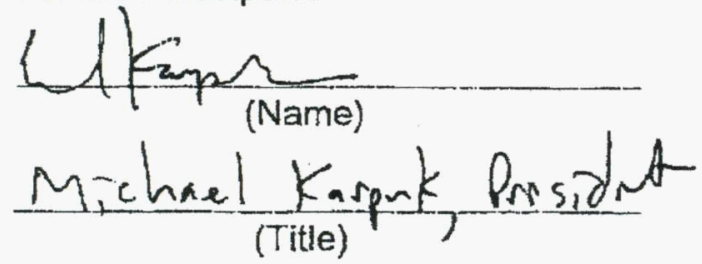

$\frac{\$ 28 / 07}{\text { (Date) }}$ 
Production of Endohedral Fullerenes by Ion Implantation

Final Report for CRADA \#ORNL 01-0624

May 31, 2007

by:

Michael D. Diener

J. Michael Alford

TDA Research, Inc.

12345 W. 52nd Ave.

Wheat Ridge, CO 80033-1916

\section{Saed Mirzadeh}

Nuclear Medicine Program

Isotope Development Group

Nuclear Science and Technology Division

Oak Ridge National Laboratory

P.O Box 2008

Oak Ridge TN, 37922-6229

For:

UT-Battelle, LLC

Director, Technology Transfer Office

APPROVED FOR PUBLIC RELEASE 


\begin{abstract}
The empty interior cavity of fullerenes has long been touted for containment of radionuclides during in vivo transport, during radioimmunotherapy (RIT) and radioimaging for example. As the chemistry required to open a hole in fullerene is complex and exceedingly unlikely to occur in vivo, and the conformational stability of the fullerene cage is absolute, atoms trapped within fullerenes can only be released during extremely energetic events. Encapsulating radionuclides in fullerenes could therefore potentially eliminate undesired toxicity resulting from leakage and catabolism of radionuclides administered with other techniques. A the start of this project however, methods for production of transition metal and p-electron metal endohedral fullerenes were completely unknown, and only one method for production of endohedral radiofullerenes was known. We therefore investigated three different methods for the production of therapeutically useful endohedral metallofullerenes: 1) implantation of ions using the high intensity ion beam at the Oak Ridge National Laboratory (ORNL) Surface Modification and Characterization Research Center (SMAC) and fullerenes as the target; 2) Implantation of ions using the recoil energy following alpha decay; and 3) implantation of ions using the recoil energy following neutron capture, using ORNL's High Flux Isotope Reactor (HFIR) as a thermal neutron source. While we were unable to obtain evidence of successful implantation using the ion beam at SMAC, recoil following alpha decay and neutron capture were both found to be economically viable methods for the production of therapeutically useful radiofullerenes. In this report, the procedures for preparing fullerenes containing the isotopes ${ }^{212} \mathrm{~Pb},{ }^{212} \mathrm{Bi},{ }^{213} \mathrm{Bi}$, and ${ }^{177} \mathrm{Lu}$ are described. None of these endohedral fullerenes had ever previously been prepared, and all of these radioisotopes are actively under investigation for RIT. Additionally, the chemistry for derivatizing the radiofullerenes for water-solubility and a method for removing exohedral radionuclides are reported. The methods and chemistry developed during this CRADA are the crucial first steps for the development of fullerenes as a method superior to existing technologies for in vivo transport of radionuclides.
\end{abstract}




\section{Table of Contents}

1. Introduction $1-2$

2. Ion Implantation at SMAC 2

3. Implantation by Recoil Following Alpha Decay 2-7

3.1. Implantation by Recoil Following Alpha Decay 5

3.1.1 Radioactivity Measurements 5

3.1.2 Early Work; Ion Implantation 5-6

3.1.3 Coating Radionuclides with Fullerenes; Recoil Implantation 6-7

3.2. Derivatization and Purification of $\mathrm{R} @ \mathrm{C}_{60} \quad 7-15$

3.2.1 Esterification of $\mathrm{R} @ \mathrm{C}_{60}$; Washing of the Esters 8-10

3.2.2. Control Experiment: Washing of Esters $10-11$

With Very Short Contact Time 11

3.2.3. Direct Recoil into Esters 11-12

3.2.4. The Fate of the ${ }^{212} \mathrm{Bi} F r o m{ }^{212} \mathrm{~Pb} @ \mathrm{C}_{60}$ Malonic Ester Derivatives 12-13

3.2.5. Conversion of the Ester to the Acid 13-15

3.3. Mouse Biodistributions of ${ }^{212} \mathrm{~Pb} @ \mathrm{C}_{60}\left(\mathrm{C}\left[\mathrm{CO}_{2}{ }^{-}\right]_{2}\right)_{\mathrm{x}}$

3.3.1. One Hour Study 15-16

3.3.2. Three Hour and Eight Hour Biodistributions 16-17

3.3.3. Average Biodistribution; Comparison to Previous Studies 17-19

3.4. Summary of ${ }^{212} \mathrm{~Pb}$ Studies 19

4. Implantation of ${ }^{177} \mathrm{Lu}$ by Recoil Following Neutron Capture 19-35

4.1. Theoretical Study for Production of ${ }^{177} \mathrm{Lu} @ \mathrm{C}_{60} \quad 20-21$

4.2. Neutron Activation to ${ }^{177} \mathrm{Lu}$ in Mixtures of Lutetium Oxide and $\mathrm{C}_{60}$

4.2.1. Neutrons From the HFIR 22-23

4.2.2. Irradiation of $\mathrm{Natural} \mathrm{Lu}_{2} \mathrm{O}_{3}-\mathrm{C}_{60}$ Mixtures 23-24

4.2.3. Activation of ${ }^{176} \mathrm{Lu}_{2} \mathrm{O}_{3}-\mathrm{C}_{60}$ Mixtures 24

4.3. Assessment of the Recoil Formation Yield of ${ }^{177} \mathrm{Lu} @ \mathrm{C}_{60}$

4.3.1. Results of Malonic Ester Derivatization and Washing 25

4.3.2. The Fundamental Problem with Recoil Formation of

Radiofullerenes From Mixtures With Metal Oxides 26-27

4.3.3. Avoiding the Fundamental Problem: Preparation and

Activation of Non-oxide $\mathrm{Lu}-\mathrm{C}_{60}$ Mixtures $\quad 27-29$

4.4 HPLC Separation of ${ }^{177} \mathrm{Lu} @ \mathrm{C}_{60}$ Bingel Ester from Cold $\mathrm{C}_{60}$ Bingel Esters 29-34

4.5 Summary of ${ }^{177} \mathrm{Lu} @ \mathrm{C}_{60}$ Studies. 34

References

35-38 


\section{List of Tables}

Table 1: Activity of select radionuclides remaining on the electroplated $\mathrm{Pt}$ meshes after $\mathrm{C}_{60}$ was removed by base-catalyzed chemical derivatization (see next section for detail).

\section{List of Figures}

Figure $1 . \mathrm{C}_{60}$ containing an encapsulated atom

Figure 2: The major decay pathways for generating therapeutically useful $\alpha$-emitting radionnuclides

Figure 3: Cell for implantation of ions following $\alpha$-decay into fullerenes

Figure 4: Processing of the fullerene esters. The last three steps are repeated as necessary, typically six or seven times.

Figure 5: Successive washings of ${ }^{212} \mathrm{~Pb} @ \mathrm{C}_{60}$ malonic ester derivatives in ethyl acetate with dilute nitric acid containing cold lead atoms. Removal of atoms not recoiled into the $\mathrm{C}_{60}$ cage is also demonstrated.

Figure 6: Successive washings of ${ }^{213} \mathrm{Bi} @ \mathrm{C}_{60}$ malonic ester derivatives in ethyl acetate with dilute nitric acid containing cold bismuth atoms. "Calculated" is how much ${ }^{213} \mathrm{Bi}$ was actually inside of the fullerene at the times the washings were performed, back calculated from the $33 \mathrm{nCi}$ that did not wash out during the last wash.

Figure 7: Successive washings of ${ }^{212} \mathrm{~Pb} @ \mathrm{C}_{60}$ malonic ester derivatives in ethyl acetate with dilute nitric acid containing cold lead atoms. 25 minute contact time. Note the change in scale versus Figure 6.

Figure 8: Successive washings of ${ }^{212} \mathrm{~Pb} @ \mathrm{C}_{60}$ malonic ester derivatives in ethyl acetate with dilute nitric acid containing cold lead atoms. Removal of ${ }^{225} \mathrm{Ac}$ atoms not recoiled into the $\mathrm{C}_{60}$ cage is also demonstrated. The average time between washes is six minutes.

Figure 9: Comparison of ${ }^{212} \mathrm{Bi}$ activity during washing to that of ${ }^{212} \mathrm{~Pb}$.

Figure 10: Counting an aqueous wash of $\mathrm{C}_{60}$ esters, showing decay of the ${ }^{212} \mathrm{Bi}$ to secular equilibrium with the ${ }^{212} \mathrm{~Pb}$ in the wash.

Figure 11: Process for conversion of the ester to the carboxylic acid

Figure 12: One hour biodistribution of ${ }^{212} \mathrm{~Pb} @ \mathrm{C}_{60}\left[\mathrm{C}\left(\mathrm{COO}^{-}\right)_{2}\right]_{\mathrm{x}}$, including standard error. See text for details.

Figure 13: Three hour (left) and eight hour (right) biodistributions of ${ }^{212} \mathrm{~Pb} @ \mathrm{C}_{60}\left[\mathrm{C}\left(\mathrm{COO}^{-}\right)_{2}\right]_{\mathrm{x}}$.

Figure 14: Average biodistribution of ${ }^{212} \mathrm{~Pb} @ \mathrm{C}_{60}\left[\mathrm{C}\left(\mathrm{COO}^{-}\right)_{2}\right]_{\mathrm{x}}$ using the data presented in the previous two subsections. "Error bars" show standard deviation.

Figure 15. Kinetic model for the production of ${ }^{177} \mathrm{Lu} @ \mathrm{C}_{60}$ by recoil implosion in a nuclear reactor. Where $\mathrm{n}$ is the number of Lu nuclide in the target, and $v$ is the flux density of thermal neutron 


\section{List of Figures (cont.)}

Figure 16. Integrated rate equations yielding the total activity of ${ }^{177} \mathrm{Lu} @ \mathrm{C}_{60}$ and the specific activity of ${ }^{177} \mathrm{Lu} @ \mathrm{C}_{60}$ as a function of the irradiation time. The specific activity is calculated as the percent of

${ }^{177} \mathrm{Lu} @ \mathrm{C}_{60}$ relative to all endohedral $\mathrm{C}_{60}$ species produced.

Figure 17: PT-1 of the HFIR; PT-2 was used to neutron activate the samples, and they are similarly designed and located.

Figure 18: Gamma ray spectrum of the $3.0 \mathrm{mg} \mathrm{Lu} \mathrm{L}_{3} / \mathrm{C}_{60}$ mixture 4.5 days after irradiation for $20 \mathrm{~min}$ in PT-2.

Figure 19: Washing the bucky ester with an aqueous $\mathrm{Lu}$ - nitric acid solution. Error bars are from the uncertainty in counting data. See text for details.

Figure 20: Chart of the activity during the work-up of the $\mathrm{Lu}(\mathrm{acac})-\mathrm{C}_{60}-\mathrm{DMF}$ sample. The sum of the toluene and aqueous activities following a wash is $85-109 \%$ of the toluene solution prior to that wash.

Figure 21: Enthlapies of Sublimation for the Lanthanides (Cotton and Wilkinson, 1988).

Figure 22: Left: Absorbance at $310 \mathrm{~nm}$ of a mixture of empty $\mathrm{C}_{60}$ with 4, 5, and 6 malonic esters in toluene chromatographed on a semi-prep BuckyPrep column with $3 \mathrm{~mL} / \mathrm{min}$ each toluene and hexane. Right: UV - vis absorbance at times corresponding to the vertical lines of the same color in the chromatogram, i.e. the double-humped dark blue spectrum elutes after the single-humped light blue, which is after the red with the absorbance in the visible. The two spectra with little absorbance are the last two vertical lines in the chromatogram

Figure 23: Chromatogram of ${ }^{176} \mathrm{Lu}$ oxide $+\mathrm{C}_{60}$ mixture, derivatized by the Bingel procedure, loaded onto the BuckyPrep column, and eluted with $3 \mathrm{~mL} / \mathrm{min}$ toluene $+3 \mathrm{~mL} / \mathrm{min}$ hexane. Top: uv absorbance @, 310nm. Bottom: ${ }^{177} \mathrm{Lu}$ activity in fractions collected every 30 seconds from injection.

Figure 24: Left: Absorbance at $310 \mathrm{~nm}$ of a mixture of empty $\mathrm{C}_{60}$ with 4, 5, and 6 malonic esters in toluene chromatographed on a semi-prep BuckyPrep column with $6 \mathrm{~mL} / \mathrm{min}$ ethylacetate. Right: UV vis absorbance at times corresponding to the vertical lines of the same color in the chromatogram, i.e. the double-humped red spectrum elutes before the weak single-humped dark blue, which is before the light blue with the absorbance in the visible $(470 \mathrm{~nm})$.

Figure 25: Chromatogram of $\mathrm{Lu}(\mathrm{acac})-\mathrm{C}_{60}$ mixture, coprecipitated from $\mathrm{DMF}$, activated, derivatized by the Bingel procedure, loaded onto the BuckyPrep column, and eluted with $4 \mathrm{~mL} / \mathrm{min}$ ethyl acetate +2 $\mathrm{mL} / \mathrm{min}$ hexane. Top: uv absorbance @ 310nm. Bottom: ${ }^{177} \mathrm{Lu}$ activity in fractions collected every 30 seconds from injection. 


\section{Introduction}

Fullerenes, also known as "buckyballs," are carbon clusters which have cage-like structures. Their geodesic structures, resembling soccer balls, are formed by the sequential bonding of cyclic carbon structures. Soon after their discovery in 1985 (Kroto et al., 1985), it was recognized that a small molecule or atom could be trapped inside the empty cage (FigureFigure 1; Heath et al., 1985). Indeed, when the fullerenes are formed in the presence of certain metal ions, particularly the lanthanides, the metal atom is often trapped within the fullerene cage. For example, $\mathrm{Y} @ \mathrm{C}_{82}$ means a yttrium atom is trapped within the $\mathrm{C}_{82}$ fullerene cage. Although $\mathbf{M} @ \mathrm{C}_{60}$ represents the largest component of so-called "endohedral" fullerenes produced by typical methods, in general only $\mathrm{M} @ \mathrm{C}_{82}$ and a few select other larger fullerenes survive the purification process by solvent extraction (Diener and Alford, 1998).

Endohedral metallofullerenes have been prepared from electropositive metals like alkaline earth metals (Group 2, save magnesium) and lanthanides and uranium (Group 3). The standard technique is co-vaporization of carbon and the metal by arc discharge or laser ablation (Chai et al., 1991). So far, attempts to produce fullerenes containing transition metals by these techniques have not been successful. Noble gas endohedral fullerenes have also been prepared with very low yields by high pressure, high temperature techniques that essentially squeeze a few gas atoms into the fullerene cage (Saunders et al., 1994). Techniques based on recoil from gamma- ray emission following

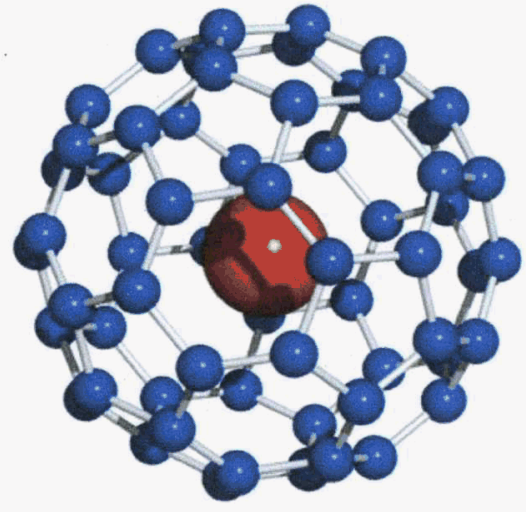

Figure 1. $\mathrm{C}_{60}$ containing an encapsulated atom. neutron capture have also been used to generate a range of endohedral fullerenes, including fullerenes containing Sb, As, and Se, but again the yield is very low (Ohtsuki et al., 2000). Light ions - specifically $\mathrm{He}^{2+}$ and $\mathrm{Li}^{+}$- have been ion implanted in fullerenes at respectable yields (Gromov et al., 2003).

It is now generally accepted that due to exceptional cage stability with respect to cage opening, combined with the ease at which the outer surfaces can be functionalized, endohedral fullerenes show great promise in a number of areas of medical diagnosis and treatment. It is envisioned that metallofullerenes could provide an alternative to chelating compounds currently used in nuclear medicine because of their resistance to metabolism, as well as their high thermodynamic and kinetic stability. Our recent work has shown that radiofullerenes can be successfully isolated, derivatized and solubilized. By irradiating prepurified ${ }^{165} \mathrm{Ho} @ \mathrm{C}_{82}$ in the ORNL High Flux Isotope Reactor (HFIR), ${ }^{166} \mathrm{Ho} @ \mathrm{C}_{82}$ was produced. Holmium-166, a beta-emitting radioisotope with a half-life of $62 \mathrm{~h}$, is of interest for therapeutic applications in nuclear medicine. The results of this pioneer study, a collaboration between ORNL, TDA Research, and Rice University, were published in the Proceedings of the National Academy of Sciences (v. 96, p. 5182).

This project began as an investigation of the high intensity ion beams available at ORNL's Surface Modification and Characterization (SMAC) Research Center. Since SMAC is capable of producing a number of transition metal ions, the project was expected to lead to the discovery of new endohedral fullerenes. The most important metal to be encapsulated was believed to be Copper. However, results from experiments there were discouraging, and shortly after SMAC was permanently shut down for lack of funding.

The project was then amended to refocus on production of radiofullerenes by recoil. During recoil implantation, the radionuclides encapsulated into the fullerene are carrier free; i.e., the activated metal is 
isolated from the non-activated metal by the fullerene, thus producing radiofullerenes with very high specific activities (a new variation of the well known Szilard-Chalmers effect). The chemical differences between the radionuclide and the metallofullerene provide a basis for rapid separation of encapsulated from unencapsulated radionuclides. The recoil implantation can also produce radiofullerenes from elements that cannot be incorporated into fullerenes by carbon vapor synthesis.

In the second series of experiments, various therapeutically useful alpha-emitting radionuclides were encapsulated by recoil following alpha-decay of a parent radionuclide. This work prepared for the first time endohedral lead $(\mathrm{Pb})$ and bismuth $(\mathrm{Bi})$ fullerenes, specifically the ${ }^{212} \mathrm{~Pb},{ }^{212} \mathrm{Bi}$, and ${ }^{213} \mathrm{Bi}$ radioisotopes, all of which are of great interest for state-of-the-art cancer therapies. Indeed, the use of recoil from alpha-decay to form endohedral fullerenes have never previously been demonstrated, and, given the high kinetic energies of the recoiling nuclei, there was ample reason to question whether it would work or not. Techniques for the rapid elimination of exohedral radioisotopes and derivatization for water solubility were also developed. Finally, a preliminary biodistriubtion study showed that, as expected, encapsulation in the fullerene prevented ${ }^{212} \mathrm{~Pb}$ from locating in the bones of mice, as it does when lost from chelators in vivo.

In a third series of experiments, ${ }^{177} \mathrm{Lu}$ endohedral fullerenes were produced by neutron activation. While at first blush, this may seem to be an obvious extension of existing work, it was found that much of the existing work on neutron activation greatly overestimated the success of the technique. ${ }^{177} \mathrm{Lu}$ is also of tremendous interest in the current development of advanced cancer therapies. Since only activated $\mathrm{Lu}$ is trapped in the fullerene, the difference between fullerene chemistry and Lu chemistry provides for an essentially instant separation of activated $\mathrm{Lu}$ from cold $\mathrm{Lu}$, thereby providing carrier-free ${ }^{177} \mathrm{Lu}$, which is otherwise obtainable only by tedious and expensive additional process steps. Separation of malonic esters of ${ }^{177} \mathrm{Lu} @ \mathrm{C}_{60}$ from empty $\mathrm{C}_{60}$ by rapid HPLC was also developed.

This final report for the TDA-ORNL CRADA documents all three series of experiments in the order in which they were performed and described in the introduction. While the original plan to produce transition metal endohedral fullerenes did not proceed successfully, a tremendous amount of exciting discoveries were made with other techniques that can essentially only be practiced at ORNL's unique radiological facilities.

\section{Ion Implantation at SMAC}

The first part of this project used the high intensity ion beam at ORNL's SMAC as a source of transition metal ions accelerated to sufficiently high energies to be implanted into $\mathrm{C}_{60}$. Although various beam currents and copper ion energies were tested, no evidence could be found for $\mathrm{Cu} @ \mathrm{C}_{60}$ by laser-desorption time-of-flight mass spectrometry (LD-TOF-MS). The detection limit for the TOF-MS instrument at that time was approximately one part in one thousand for easily ionizable copper endohedral fullerenes. It is possible that the technique was successful at lower yields, but such low yields are not of commercial interest. Following this series of experiments, we moved on to utilize other unique facilities at ORNL for the production of marketable, therapeutically useful endohedral fullerenes.

\section{Implantation by Recoil Following Alpha-Particle Decay}

The second part of this project sought to recoil the nucleus following alpha-particle decay into the cavity of $\mathrm{C}_{60}$. The daughter nucleus breaks through a few fullerenes, losing energy, until some nuclei run out of kinetic energy while they are inside of a fullerene. Since fullerenes are extremely inexpensive in comparison to alpha-emitting radionuclides, and the value of therapeutically useful radiofullerenes is expected to be quite high, a low conversion of fullerenes to endohedral fullerenes is irrelevant. However, 
the radionuclide to be encapsulated must be chosen very carefully, so as to maximize the ultimate economic value of radiofullerene.

The best use of radiofullerenes is thought to be in radioimmunotherapy (RIT; Milenic et al., 2004, e.g.). In RIT, metallic radionuclides are contained in polyaminocarboxylate (PAC) chelators, wherein three or more secondary amines and carboxylic acids chelate the radionuclide, largely sheltering it from the external environment. The chelated radionuclides are then delivered to cancer cells by chemically attaching the radionuclide to an antibody, antibody fragment, or peptide that is specific for receptors expressed solely (or at least preferentially) on the cancer cells. Following arrival of the radionuclide antibody conjugates at the cancer cell, decay products from the radionuclides can then break strands of the cell's DNA, thereby killing the cancerous cell. RIT using PAC chelators and beta-emitting radionuclides has recently become an attractive method for the treatment of B-cell non-Hodgkin's lymphoma (NHL), with two radiopharmaceuticals approved by the FDA.

Effective targeting of the radionuclides remains the primary challenge in RIT (Sharkey et al., 2004, e.g.). Current limitations in targeting efficacy restricts effective use of RIT to attack on cancerous cells that have tremendous exposure to circulating blood (and thus the circulating antibody conjugate). The haematological malignancies that can be effectively targeted frequently have a significant portion (if not a majority) of the cancerous cell volume consisting of single cells or aggregates of a few cells, tens of microns in diameter, much smaller than the distance over which $\beta$-particles distribute their energy in vivo (millimeters). Therefore, $\beta$-decays in RIT invariably primarily damage healthy tissues. Moreover, a single beta decay is insufficient to break more than one strand of DNA, and single-strand breaks can be readily repaired. Microdosimetry calculations indicate that hundreds of thousands of beta-particles are required to have a $99.99 \%$ chance of killing a cell. Conversely, only hundreds of alpha-particles are required for the same probability of killing the cell, and the range of alpha decays in vivo is $\sim 50$ microns. Although the FDA-approved NHL therapies use one of two beta-emitting radionuclides $\left({ }^{90} \mathrm{Y}\right.$ or $\left.{ }^{131} \mathrm{I}\right)$, it is widely agreed that alpha particles therefore have ideal characteristics for the treatment of the cancers that RIT can most effectively target. Furthermore, complexes of lanthanide radionuclides with existing PAC chelators appear to be sufficiently stable for use in current RIT applications, obviating the need for fullerenes.

Despite the favorable properties of $\alpha$-particle radiation, development of $\alpha$-particle RIT has been limited by the poor availability and/or physical characteristics of $\alpha$-emitting radionuclides. These intrinsic drawbacks have limited development of $\alpha$-emitters, while focusing attention on $\beta$-emmiters. (One potential use of fullerenes in beta-particle RIT is given in Section 4.) Several reviews have debated the relative strengths and weaknesses of the available $\alpha$-emitting radionuclides (Couturier et al., 2005; Mulford et al., 2004; Zalutsky and Pozzi, 2004; Mirzadeh, 1998). Currently, most effort is focused on ${ }^{211} \mathrm{At},{ }^{225} \mathrm{Ac}$ and ${ }^{213} \mathrm{Bi}$. The decay characteristics of ${ }^{211} \mathrm{At}$ are very attractive (single decay to ground state, $\mathrm{t}_{1 / 2}=7.2 \mathrm{~h}$ ), but, using current state-of-the-art production methods (Zalutsky et al., 2001), the creation of one human dose of ${ }^{211}$ At requires about one day of operation at an unusually large cyclotron, and is therefore most likely to be prohibitively expensive for the foreseeable future. ${ }^{225} \mathrm{Ac}\left(\mathrm{t}_{1 / 2}=10\right.$ days) is readily available from stockpiled ${ }^{233} \mathrm{U}$, and generator systems have been developed for its daughter ${ }^{213} \mathrm{Bi}$ $\left(t_{1 / 2}=45 \mathrm{~min}\right)$. While both of these radionuclides can be satisfactorily chelated by a PAC, the four $\alpha-$ emitting daughters of ${ }^{225} \mathrm{Ac}$ (Figure 2) are not retained in the PAC and are free to redistribute. A recent primate study demonstrated dose-limiting renal toxicity, believed to result from free ${ }^{213} \mathrm{Bi}$ decays (Miederer et al., 2004). So far, none of the approaches to corral the daughters have been successful (Jaggi et al., 2005), and given the high recoil energy of $\alpha$-decays, it is also very unlikely that fullerenes could be of assistance. While ${ }^{213} \mathrm{Bi}$ decays directly to a stable isotope, its short half life requires more efficient targeting than is currently available (Adams et al., 2000). 

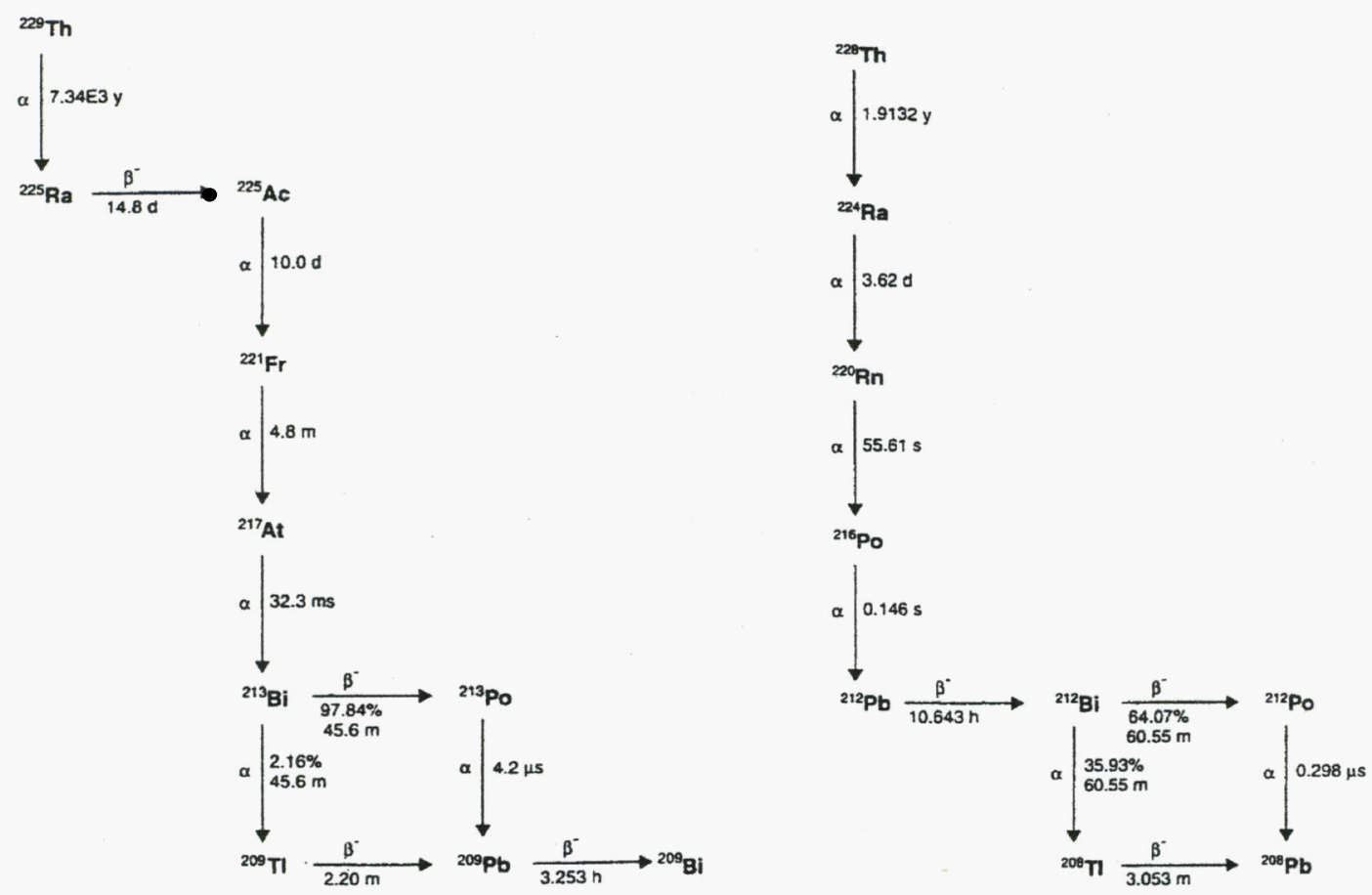

Figure 2: The major decay pathways for generating therapeutically useful $\alpha$-emitting radionnuclides

The original RIT studies were performed with a fourth isotope, ${ }^{212} \mathrm{Bi}$, in large part because both parent material and a generator system were readily available. The decay chain of ${ }^{228} \mathrm{Th}$, of which ${ }^{212} \mathrm{Bi}$ is a daughter, is shown in Figure 2. The half life ${ }^{212} \mathrm{Bi}$ creates the same limitations as for ${ }^{213} \mathrm{Bi}$, but that problem can potentially be overcome by using ${ }^{212} \mathrm{~Pb}$. While ${ }^{212} \mathrm{~Pb}$ is a beta emitter, it is desirable for $\alpha-$ particle RIT because its $10.6 \mathrm{~h}$ half-life increases by tenfold the time available to get ${ }^{212} \mathrm{Bi}$ to the target tissue before $\alpha$-decay. However, while DOTA and other chelators can apparently chelate ${ }^{212} \mathrm{~Pb}$ satisfactorily in saline solutions (Mirzadeh et al., 1993; Chappell et al., 2000; Chong et al., 2006), in vivo tests resulted in severe myelotoxicity, presumably the result of bone-targeting ${ }^{212} \mathrm{~Pb}^{2+}$ lost or transchelated out from the DOTA (Ruble et al., 1996; Horak et al., 1997; Chong et al., 2006). Furthermore, the internal conversion following $36 \%$ of the beta decays to ${ }^{212} \mathrm{Bi}$ also released the daughter from the DOTA. The use of a fullerene instead of a chelator could potentially solve both problems with ${ }^{212} \mathrm{~Pb}$, thereby making the ${ }^{212} \mathrm{~Pb} /{ }^{212} \mathrm{Bi}$ system attractive for RIT. If the fullerene was successful, the only significant remaining drawback to its use is the high-energy gamma-ray emitted by the ${ }^{209} \mathrm{Tl}$ daughter. Yet, compared to the outstanding problems with ${ }^{225} \mathrm{Ac}$ and ${ }^{213} \mathrm{Bi}$, solutions for shielding health care personnel from high-energy gamma-rays are well known, and inexpensive compared to the costs of ${ }^{211}$ At production.

Herein is reported the synthesis of ${ }^{212} \mathrm{~Pb} @ \mathrm{C}_{60}$ by recoil following $\alpha$-decay of its short-lived parent (Section 3.1), the formation of water-soluble ${ }^{212} \mathrm{~Pb} @ \mathrm{C}_{60}$ malonic acids (Section 3.2.1), the stability of the radiofullerene during beta-decay (Section 3.2.2), and a preliminary biodistribution study of the untargeted water-soluble radiofullerene in mice (Section 3.3). To our knowledge, this is the first endohedral fullerene formed by recoil from $\alpha$-decay, the first endohedral lead fullerene, the first fullerene encapsulating an $\alpha$ emitting radionuclide with a therapeutically useful half-life, and the first biodistribution study of fullerene malonic acids. Since we began this work, two reports of new chelators for ${ }^{212} \mathrm{~Pb}$ have appeared, but the results appear to indicate that further development or a new approach (i.e., fullerenes) is required. 


\subsection{Implantation by Recoil Following Alpha Decay}

\subsubsection{Radioactivity Measurements}

Since $\alpha$-particles do not penetrate very far into a dense medium, it is more common to assess the presence of the $\alpha$-emitting radionuclide by measuring the number of gamma ray(s) emitted as part of the $\alpha$-decay processes. The gamma rays are measured by a cooled germanium detector The instrument had previously been calibrated for detector efficiency as a function of both the photon energy and distance between the sample and the detector. The calibration factor was then multiplied by the probability of the photon being emitting during the decay, as given in a table of the isotopes. The resolution of the instrument as operated was $0.5 \mathrm{keV}$. Gamma rays were typically observed between 10 and $1000 \mathrm{keV}$. A software program then calculated the area under the peaks corresponding to emitted photons. The peak areas were then converted to the activity of each component of the sample using the calibration factor and photon probability product.

Since the probability of a certain photon being emitted varies widely according to the photon, it can be advantageous to track the presence of certain isotopes through their daughters. For example, ${ }^{225} \mathrm{Ac}$ emits no photons with a probability much greater than $1 \%$, while the $217 \mathrm{keV}$ and $440 \mathrm{keV}$ photons from ${ }^{221} \mathrm{Fr}$ and ${ }^{213} \mathrm{Bi}$ are emitted 12 and $26 \%$ of the time, respectively. Thus, the short-lived daughters of ${ }^{225} \mathrm{Ac},{ }^{221} \mathrm{Fr}$ and/or ${ }^{213} \mathrm{Bi}$ are typically used to track the presence of ${ }^{225} \mathrm{Ac}$ in this study. Similarly, ${ }^{212} \mathrm{Bi}$ can be tracked much more effectively via the ${ }^{208} \mathrm{Tl} 583 \mathrm{keV}$ photon emitted $86 \%$ of the time (using the appropriate correction factor), than with its own weak $727 \mathrm{eV}$ gamma ray. ${ }^{224} \mathrm{Ra}$ can be tracked via its $241 \mathrm{keV}$ photon. Generally, collecting data for five minutes at the appropriate distance to the detector was sufficient to assess the radionuclide content of the sample down to sub-nanocurie levels.

3.1.2 Early Work; Ion Implantation When this project was started, only three methods were known for encapsulating atoms in fullerenes: 1) During the formation of the fullerene by condensation of carbon (and metal) vapor (Chai et al., 1991);2) Under high pressures and temperatures of the atom to be encapsulated (Saunders et al., 1996); 3) During recoil following thermal neutron capture (Rausch and Braun, 2001). Since therapeutic $\alpha$-emitting radionuclides are, generally speaking, not produced by neutron activation, the third method was not relevant. High pressure methods are typically useful only for gases, such as helium, and the yields are very low.

However, ORNL team found a simpler, cleaner method while this proposal was under review. In this method, ${ }^{225} \mathrm{Ac}$ was He electroplated onto one disk, coated a second disk with $\mathrm{C}_{60}$, applied a a potential of a few $\mathrm{kV}$ between the two disks (electrodes), and within a few hours ${ }^{213} \mathrm{Bi} @ \mathrm{C}_{60}$ was found on the $\mathrm{C}_{60}$ disk. During $\alpha$-decay of ${ }^{225} \mathrm{Ac}$, the Francium daughter possesses significant kinetic energy, and for a very short period of time $\left(\sim 10^{-12} \mathrm{~s}\right)$ it is very highly charged. In the presence of the electric field, the ${ }^{221} \mathrm{Fr}$ ions are accelerated across the gap and, absent of any obstacle, are implanted either in the fullerene lattice, or in the fullerenes themselves. If they are in the lattice, the recoil from a subsequent decay (of ${ }^{217} \mathrm{At}$ ) can implant ${ }^{213} \mathrm{Bi}$ in $\mathrm{C}_{60}$. The ORNL team regularly found $\sim 20 \%$ of the ${ }^{213} \mathrm{Bi}$ expected to have formed during the time the disks faced each other on the $\mathrm{C}_{60}$ disk, with $1-2 \%$ encapsulated.

There are significant advantages of this system over the co-vaporization ( $\operatorname{arc}$ ) method. Primarily, the parent radionuclide remains sequestered on the disk that does not contain fullerenes and does not require handling, allowing it to serve as a generator for future generation of daughters. Also, the equipment is simpler, with no moving parts to be operated in a glove box, nor generation of high temperature, nor need to confine radioactive dust. 
However, to encapsulate ${ }^{225} \mathrm{Ac}$ in this method requires starting from ${ }^{229} \mathrm{Th}$, and implanting ${ }^{225} \mathrm{Ra}$ into the $\mathrm{C}_{60}$ as it comes across the gap. ${ }^{225} \mathrm{Ra}$ then $\beta$-decays to ${ }^{225} \mathrm{Ac}$, and the $\beta$-decay is not strong enough to rupture the fullerene cage.) Typically, atoms require $\sim 120 \mathrm{eV}$ before they can be encapsulated in a $\mathrm{C}_{60}$. We calculated that a moderate vacuum and a potential of only tens of volts should be sufficient to accelerate the ${ }^{225} \mathrm{Ra}$ to at least $120 \mathrm{eV}$. A drawing of the cell used for these experiments is shown in Figure 3.

Despite meeting these conditions, it was never demonstrated that any ${ }^{225} \mathrm{Ac}$ was encapsulated by this method. One problem was simply getting the daughters to cross the gap between the electrodes. When Dr. Mirzadeh starts from purified ${ }^{225} \mathrm{Ac}, \sim 30 \%$ of the ${ }^{213} \mathrm{Bi}$ produced during the time which a potential is applied between the electrodes can be found on the fullerene disk. Similarly, more optimized systems can get $60 \%$ of the ${ }^{213} \mathrm{Bi}$ daughter across. We typically found only $\sim 1 \%$ of ${ }^{213} \mathrm{Bi}$

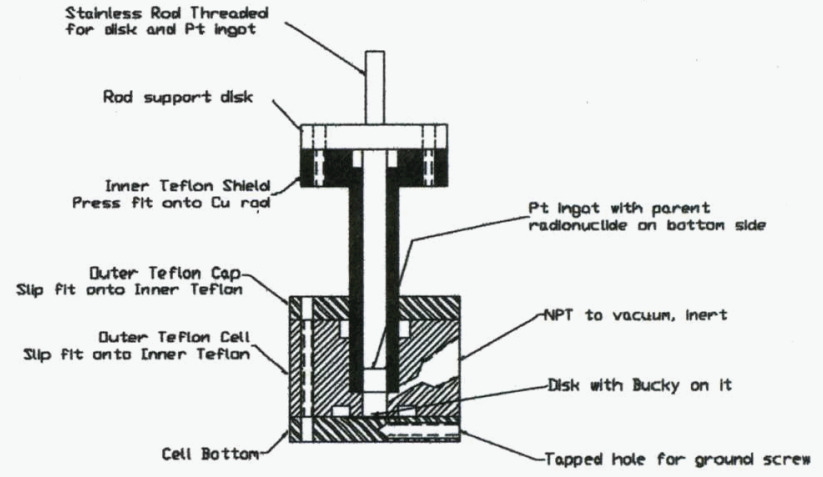

Figure 3: Cell for implantation of ions following $\alpha$-decay into fullerenes on the fullerene disk, and no obvious ${ }^{225} \mathrm{Ac}$. It is believed that the presence of significant amounts of natural thorium ${ }^{232} \mathrm{Th}$ in the ${ }^{228 / 229} \mathrm{Th}$ mixture resulted in an electroplated film several microns thick, preventing the daughters from escaping the surface and into the electrostatic field. While we considered using the incredibly expensive highly pure ${ }^{229} \mathrm{Th}(\sim \$ 40 / \mu \mathrm{Ci})$, it also occurred to us that it would be less expensive to use mixed material, even if it could not be recycled.

\subsubsection{Coating Radionuclides with Fullerenes; Recoil Implantation}

For example, from $1 \mathrm{mCi}$ of ${ }^{224} \mathrm{Ra}$, which was extractable from an existing ${ }^{228 / 229} \mathrm{Th}$ source at ORNL, we could generate more than enough ${ }^{212} \mathrm{~Pb}$ for use in mouse biodistribution studies. The ${ }^{224} \mathrm{Ra}$ has no materials cost associated with it, as it is usually left to decay while the ${ }^{225} \mathrm{Ac}$ from the Th source is processed for sale. Thus we incurred only labor costs for the generation of a mixed ${ }^{224 / 225} \mathrm{Ra}$ material. The process was expected to perform as

$$
\begin{gathered}
1 \mathrm{mCi}^{224} \mathrm{Ra} \Rightarrow 500 \mu \mathrm{Ci}^{224} \mathrm{Ra} \text { electroplated } \Rightarrow 5 \mu \mathrm{Ci}^{212} \mathrm{~Pb} @ \mathrm{C}_{60} \text { via recoil implantation } \\
\Rightarrow 2-3 \mu \mathrm{Ci} \text { water-soluble }{ }^{212} \mathrm{~Pb} @ \mathrm{C}_{60} \text { derivatives. }
\end{gathered}
$$

The system we ultimately decided on for producing ${ }^{212} \mathrm{~Pb} @ \mathrm{C}_{60}$ was incredibly simple, even simpler than that shown in Figure 3Figure . A mixture of ${ }^{224 / 225} \mathrm{Ra}$ was electroplated onto a platinum mesh. A coating of $\mathrm{C}_{60}$ was applied by spraying a toluene solution of $\mathrm{C}_{60}$ over the mesh with an artist's airbrush. As the Ra atoms and their daughters decay, some atoms are recoiled into $\mathrm{C}_{60}$ molecules. In the case of ${ }^{224} \mathrm{Ra}$, the ${ }^{220} \mathrm{Rn}$ (a noble gas) daughter may assist the process by diffusion and redistributing into the microscopic cracks in the $\mathrm{C}_{60}$ coating. Because $\mathrm{M} @ \mathrm{C}_{60}$ are generally insoluble, we removed the fullerenes (both $\mathrm{C}_{60}$ and $\mathrm{R} @ \mathrm{C}_{60}$ ) from the mesh by derivatizing them to their malonic ester derivatives, which are then soluble in tetrahydrofuran (THF), whereas underivatized fullerenes are not. The chemistry is described in the next subsection. Using this method, we were able to obtain $0.1 \%-0.5 \%$ of the ${ }^{212} \mathrm{~Pb}$ produced during the contact time as endohedral ${ }^{212} \mathrm{~Pb} @ \mathrm{C}_{60}$ derivatives. While not quite as high as hoped for, the coarseness of the preparation method can undoubtedly be improved, and the encapsulation efficiency significantly increased.

Importantly, the fullerenes could be stripped off without removing the Ra from Pt electrode (Table 1). This process is sensitive to the reaction conditions for fullerene esterification, but even during this Phase I, only a few percent of the parent radionuclides were removed from the source mesh. Many films of 
fullerenes could therefore be applied and removed from a single radionuclide source. Thus, instead of obtaining only $\sim 1 \%$ of the daughters from the parent in a fullerene, many applications of $\mathrm{C}_{60}$, and subsequent removal by esterification, allows multiple collections (each with $\sim 1 \%$ encapsulation efficiency). In the example above, $500 \mu \mathrm{Ci}$ of ${ }^{224} \mathrm{Ra}$ could generate tens of $\mu \mathrm{Ci}$ of ${ }^{212} \mathrm{~Pb} @ \mathrm{C}_{60}$ derivatives instead of only $5 \mu \mathrm{Ci}$.

Table 1: Activity of select radionuclides remaining on the electroplated Pt meshes after $\mathrm{C}_{60}$ was removed by base-catalyzed chemical derivatization (see next section for detail).

\begin{tabular}{|c|c|c|c|c|c|c|}
\hline Mesh \# and Isotope & $\# 1:{ }^{224} \mathrm{Ra}$ & $\# 1:{ }^{225} \mathrm{Ac}$ & \#2: ${ }^{224} \mathrm{Ra}$ & \#2: ${ }^{225} \mathrm{Ra}$ & $\# 3:{ }^{224} \mathrm{Ra}$ & \#3: ${ }^{225} \mathrm{Ra}$ \\
\hline Activity at first time point & 51.9 & 226 & 102 & 119 & 33.1 & 43.3 \\
\hline $\begin{array}{l}\text { Activity after fullerene } \\
\text { removal (much later } \\
\text { time) }\end{array}$ & 42.5 & 181 & 41.6 & 74.1 & 20.0 & 39.7 \\
\hline $\begin{array}{l}\text { Expected activity after } \\
\text { fullerene removal }\end{array}$ & 45.2 & 214 & 58.8 & 104 & 21.7 & 39.2 \\
\hline Radionuclide Stability & $94 \%$ & $85 \%$ & $71 \%$ & $71 \%$ & $92 \%$ & $101 \%$ \\
\hline
\end{tabular}

Most importantly, even when starting from this large mixture of radioisotopes, a combination of factors result in only ${ }^{212} \mathrm{~Pb}$ and no other radionuclides being present in the $\mathrm{C}_{60}$ by the time the chemical derivatization is finished. Thus, although the "uptake" of radionuclides into the fullerene is comparatively low versus chelators, the uptake process discriminates between radionuclides and obviates the sometimes extensive ion chromatography necessary to produce the very pure radioisotopes required by chelators. High ${ }^{212} \mathrm{~Pb}$ radiolytic purity is obtained intrinsically by the implantation process, even when starting from $\mathrm{a}^{224 / 225} \mathrm{Ra}$ generator. These results are explained further and substantiated in the following subsection.

\subsection{Derivatization and Purification of R@C $\mathbf{C}_{60}$}

The process used in this Phase I project started from a Platinum mesh and an aqueous solution of ${ }^{224 / 225} \mathrm{Ra}$ (and their daughters). The radionuclides were electroplated onto the mesh. Then a saturated toluene solution of $\mathrm{C}_{60}$ was air brushed onto the radionuclide-coated mesh, and left to stand, typically for $24-36$ hours. This section covers the processing to generate water-soluble $\mathrm{R} @ \mathrm{C}_{60}$ from a fullerene-coated mesh.

3.2.1 Esterification of $\mathbf{R} @ \mathbf{C}_{60}$; Washing of the Esters One of the most versatile and basic reactions of fullerenes is the addition of malonic esters (Figure 4). This reaction consecutively adds malonic acid esters to the fullerene cage. On empty fullerenes, the reaction proceeds through addition of the carbanion from diethylbromomalonate to $\mathrm{C}_{60}$ with $\sim 65 \%$ yield (Hirsch et al. 1993). Deprotonation of the $\alpha$-halo ester can be accomplished with $\mathrm{NaH}$ or hindered bases such as or 1,8-diazobicyclo[5.4.0]undec-7-ene (DBU). The current trend in fullerene chemistry has been to use DBU as the base. However, our early experiments showed that DBU irreversibly complexed with the metallofullerene rather than deprotonating the $\alpha$-hydrogen of the malonic ester. 


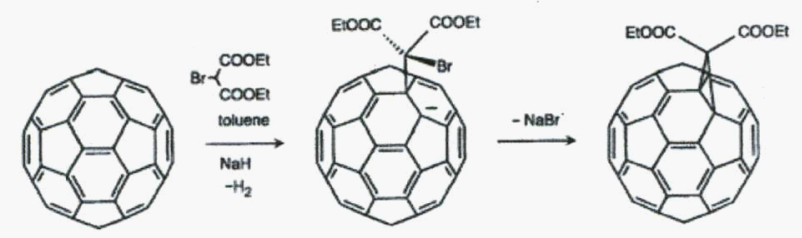

Figure 4. Malonic ester reaction sequence.

As discovered in our earlier work (Diener and Alford 1998), the metallofullerenes' redox and chemical properties are not quite identical to empty fullerenes. Metallofullerenes are much easier to reduce than empty fullerenes, explaining their tendency to complex with the lone pair of electrons on the DBU. Similarly, the basic hydride anion (from $\mathrm{NaH}$ ) also easily reduces the metallofullerene. In this case though, either hydrogen and a reduced metallofullerene or a hydrometallofullerene anion is formed. These do not block the reaction and both are soluble in the proper solvents such as THF or ethyl acetate (EA). The result is a metallofullerene substituted with three to ten malonic esters (Bolskar et al., 2001). Additional reactions hydrolyze the esters to carboxylic acids, imparting water solubility to the metallofullerene (Section 2.2.5).

The pathway for processing the $\mathrm{C}_{60}$ film into ethyl acetate (EA)soluble malonic ester derivatives is shown in Figure 4. The entire processing, including the multiple washing steps, can be performed in about an hour, although taking time to count every wash increases the time to about two hours. Sometimes the THF was transferred to the centrifuge tube and then centrifuged once, separated from the solids by transfer to a new tube, and then evaporated; when the ethyl acetate is introduced amongst those steps does not appear to have any effect on the process. The total organic volume was typically about one milliliter. Transferring was performed with syringes with stainless steel needles, typically 27 gauge. The organic solution has a characteristic deep reddish-brown color, in contrast to the purple color of pure $\mathrm{C}_{60}$.

The washing solution was made up with $10 \mathrm{~mL}$ of $0.012 \mathrm{M} \mathrm{HNO}_{3}$ containing $10 \mu \mathrm{L}$ of $1.000 \mathrm{mg} / \mathrm{mL}$ atomic absorption standards of $\mathrm{Bi}$, $\mathrm{Pb}$, and $\mathrm{Tl}$. Typically, half a milliliter of the washing solution was pipetted into the centrifuge tube containing the EA. Vortexing was usually performed for 10 seconds at $\sim 2000 \mathrm{rpm}$. Radioactive metal atoms outside of the fullerene exchange and equilibrate with the cold metal atoms present in the washing solution. Since there are so many more cold metal atoms than radioactive ones, the radioactive atoms tend to be washed out. Unless, of course, they are inside of a fullerene ester (which is not soluble in water), where they cannot exchange with the others. The esters can in principle be dissolved in

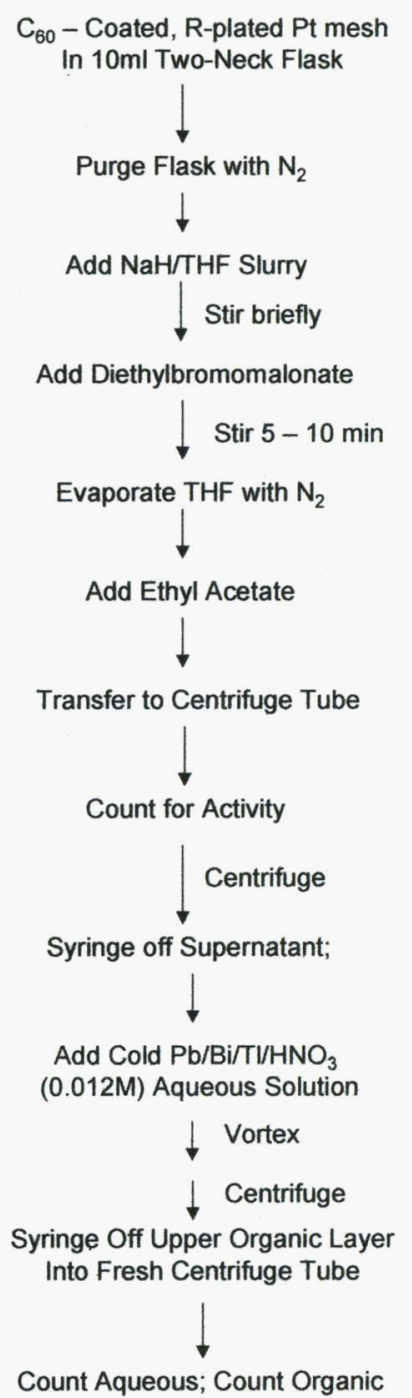

Figure 4: Processing of the fullerene esters. The last three steps are repeated as necessary, typically six or seven times. 
toluene, and then washed, but it seems to take longer to wash out the exohedral radionuclides. Note that this procedure will exchange radioactive metal atoms from chelators, as well as other more weakly chemically bonded systems.

A typical washing sequence is shown in Figure 5Error! Reference source not found.. The presence of ${ }^{212} \mathrm{~Pb}$ is tracked by the $238 \mathrm{keV} \gamma$-ray, and ${ }^{225} \mathrm{Ac}$ by the $218 \mathrm{keV} \gamma$-ray from $4.8-\mathrm{m}{ }^{221} \mathrm{Fr}$ daughter, as described on page 6. Data are not time-corrected, but the washings were performed in much less than the 10.6 hour half life of ${ }^{212} \mathrm{~Pb}$ (or the 10 day half life of ${ }^{225} \mathrm{Ac}$ ). The radioactive lead atoms outside of the fullerene are exchanged and removed in the aqueous portions, while the endohedral ${ }^{212} \mathrm{~Pb}$ atoms remain in solution. Since beta decay from ${ }^{225} \mathrm{Ra}$ is not energetic enough to implant ${ }^{225} \mathrm{Ac}$ in a fullerene, all ${ }^{225} \mathrm{Ac}$ is outside of the cage. Figure 5 shows that all of the $4.5 \mu \mathrm{Ci}$ of ${ }^{225} \mathrm{Ac}$ that were on this mesh can be separated from those radionuclides that were recoiled into the fullerene with just a few washes.

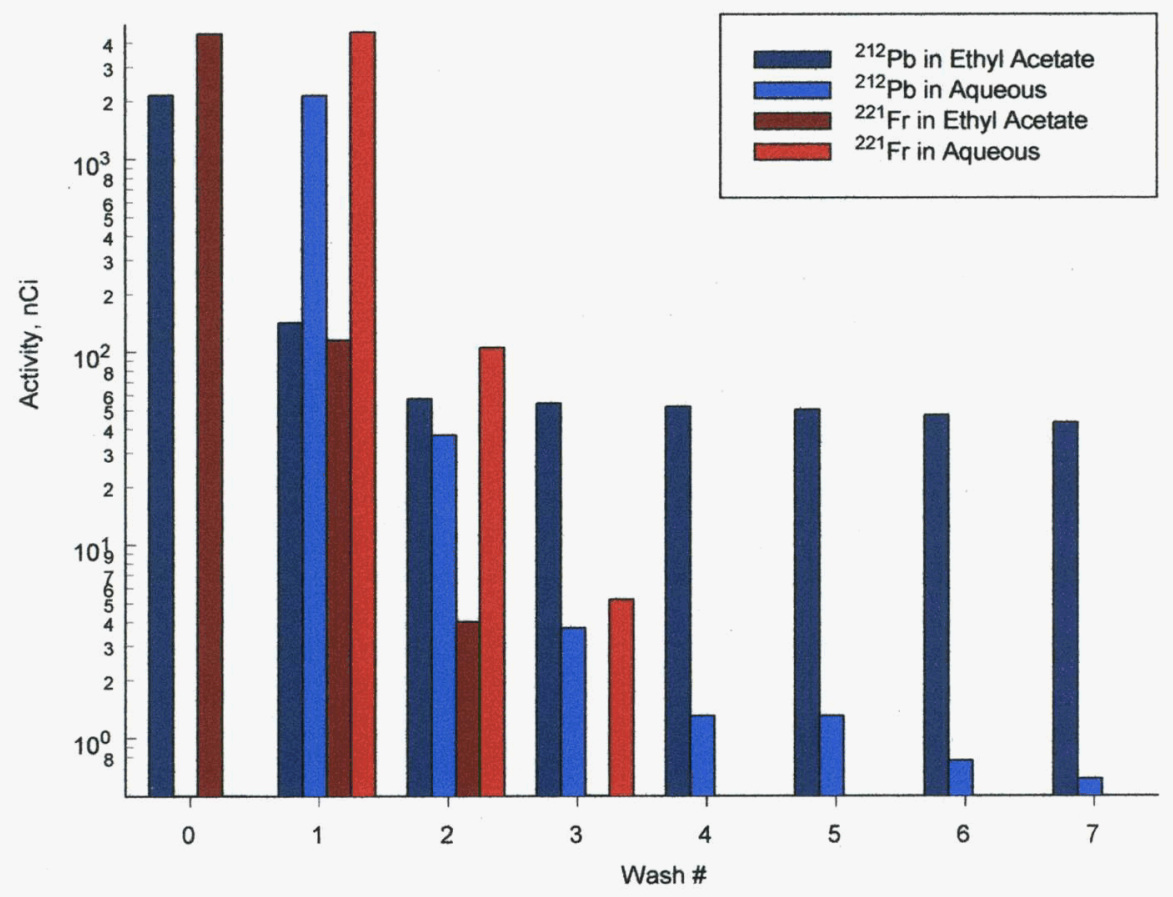

Figure 5: Successive washings of ${ }^{212} \mathrm{~Pb} @ \mathrm{C}_{60}$ malonic ester derivatives in ethyl acetate with dilute nitric acid containing cold lead atoms. Removal of atoms not recoiled into the $\mathrm{C}_{60}$ cage is also demonstrated.

${ }^{213} \mathrm{Bi}$, however, can be recoiled into $\mathrm{C}_{60}$, as shown in Figure 6. Because of its short (45.6 min) half life, the endohedral ${ }^{213} \mathrm{Bi}$ left at the time of the $7^{\text {th }}$ wash was used to back calculate the amount of ${ }^{213} \mathrm{Bi}$ present when the fullerene was removed from contact with the mesh (by esterification). In this case, the further preparation of the fullerene for water solubility took long enough that the ${ }^{213} \mathrm{Bi}$ was essentially decayed by the time the fullerenes were ready to be administered to the mice. As discussed in Section 2.2.4, several shortcuts in the synthesis appear possible, perhaps creating an opportunity for administering a combination of medium and short half-life radionuclides with a single preparation.

The encapsulation efficiency can be calculated from the washing data. For example, $150 \mu \mathrm{Ci}$ of ${ }^{224} \mathrm{Ra}$ was electroplated onto a certain Pt mesh. The fullerenes then coated it for 18 hours. The amount of ${ }^{212} \mathrm{~Pb}$ created during this time can be readily approximated using the relation $A_{D}=A_{P}\left[1-\exp \left(-.693 * t / \tau_{1 / 2 D}\right)\right]$, where $A_{D}$ is the activity of the daughter $\left({ }^{212} \mathrm{~Pb}\right.$, e.g. $), A_{P}$ is the activity of the parent $(150 \mu \mathrm{Ci}), t$ is the 
time (18 $\mathrm{h}$ in this case), and $\tau_{1 / 2 \mathrm{D}}$ is the half-life of the daughter $\left(10.6 \mathrm{~h}\right.$ for $\left.{ }^{212} \mathrm{~Pb}\right)$. The equation solves to be $104 \mu \mathrm{Ci}$.

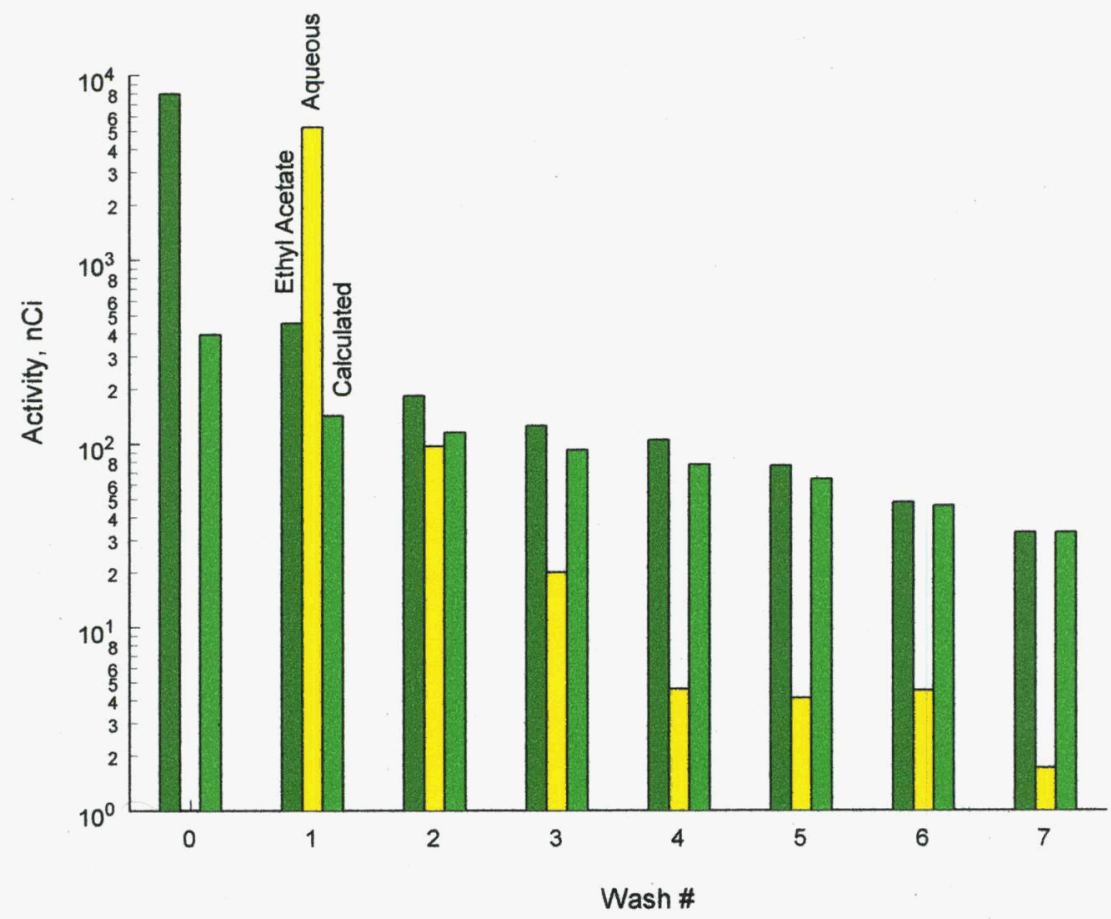

Figure 6: Successive washings of ${ }^{213} \mathrm{Bi} @ \mathrm{C}_{60}$ malonic ester derivatives in ethyl acetate with dilute nitric acid containing cold bismuth atoms. "Calculated" is how much ${ }^{213} \mathrm{Bi}$ was actually inside of the fullerene at the times the washings were performed, back calculated from the $33 \mathrm{nCi}$ that did not wash out during the last wash.

In this case, there were $220 \mathrm{nCi}$ of ${ }^{212} \mathrm{~Pb} @ \mathrm{C}_{60}$ esters in the $\mathrm{EA}$ after exhaustive washings. The derivatization and washing were performed over $6.5 \mathrm{~h}$ (other experiments were in progress simultaneously). Using $A=A_{0}\left[\exp \left(.693 * t / \tau_{1 / 2}\right)\right]$ with $A_{0}$ being the activity at the end of the contact period, $A=220 \mathrm{nCi}, \mathrm{t}=6.5 \mathrm{~h}$, and $\tau_{1 / 2}=10.6 \mathrm{~h}$, it is found that $\mathrm{A}_{\mathrm{o}}=336 \mathrm{nCi}$. The encapsuation efficiency is therefore $0.336 / 104=0.32 \%$. Using the same method, we obtained a range of values between $0.1 \%$ and $0.5 \%$, presumably varying according to the $\mathrm{C}_{60}$ film quality and the purity of the electroplated material. By preparing more uniform films of both the parent radionuclide and the fullerene, it should be possible to increase the efficiency to $1-2 \%$, based on analogy to implantation of lanthanides into fullerenes following neutron activation.

3.2.2 Control Experiment: Washing of Esters With Very Short Contact Time

To ensure that the radionuclide signals from the EA after exhaustive washing are from the fullerene ester, a control experiment was performed. A mesh covered with $51.9 \mu \mathrm{Ci}^{224} \mathrm{Ra}, 38.3 \mu \mathrm{Ci}^{212} \mathrm{~Pb}, 47.0 \mu \mathrm{Ci}^{212} \mathrm{Bi}, 62.7 \mu \mathrm{Ci}$ ${ }^{225} \mathrm{Ra}, 276 \mu \mathrm{Ci}{ }^{221} \mathrm{Fr}$, and $288 \mu \mathrm{Ci}{ }^{213} \mathrm{Bi}$ (as well as $\sim 270 \mu \mathrm{Ci}{ }^{225} \mathrm{Ac}$, presumably) was covered with $\mathrm{C}_{60}$, counted in the spectrometer to obtain those values and then put through the same ester derivatization process as before. The total contact time between the $\mathrm{C}_{60}$ and the radioactive mesh was 25 minutes. 


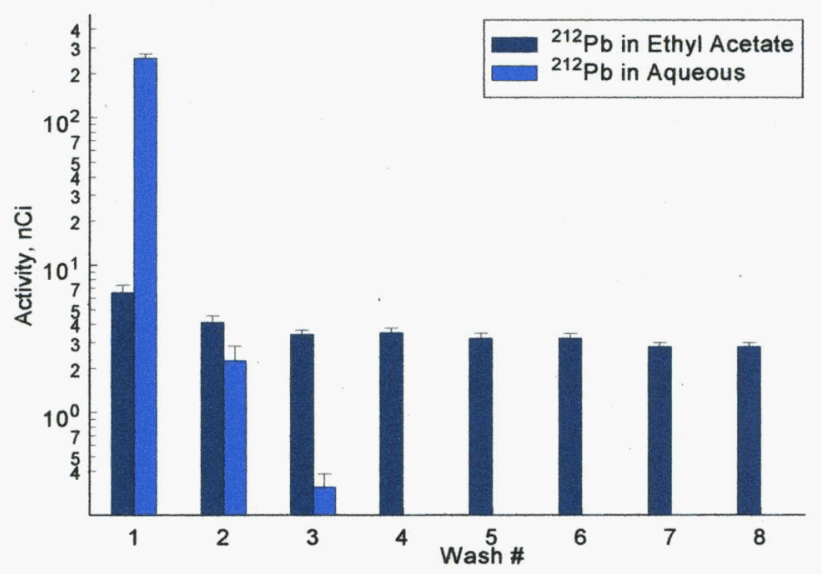

Figure 7: Successive washings of ${ }^{212} \mathrm{~Pb} @ \mathrm{C}_{60}$ malonic ester derivatives in ethyl acetate with dilute nitric acid containing cold lead atoms. 25 minute contact time. Note the change in scale versus Figure 6Error! Reference source not found..

The EA was washed exhaustively, with the ${ }^{212} \mathrm{~Pb}$ data shown in Figure 8 Figure . Clearly, some ${ }^{212} \mathrm{~Pb}$ remained in the organic, $2.8 \mathrm{nCi}$. Back-calculating gives $3.6 \mathrm{nCi}$ at the time of esterification $(3 \mathrm{~h})$. However, it was also calculated that $51.9 \mu \mathrm{Ci}$ of ${ }^{224} \mathrm{Ra}$ produces $1.38 \mu \mathrm{Ci}$ of ${ }^{212} \mathrm{~Pb}$ during 25 minutes. Thus, the encapsulation efficiency is $3.6 / 1380=0.26 \%$, very much as expected. Similar data was obtained for ${ }^{213} \mathrm{Bi}$, with $26 \mathrm{nCi}$ remaining after washing, giving $450 \mathrm{nCi}$ at the end of the contact, and 87 $\mu \mathrm{Ci}$ produced from $276 \mu \mathrm{Ci}$ of parent during the soak, for an encapsulation efficiency of $0.52 \%$. Again, this is in line with expectations.

To conclude, the amount of activity found in the organic phase after exhaustive washing with cold atoms in acid varies according to the contact time between the fullerene and the parent radionuclide in a predictable manner. The esterification chemistry does not cause the radionuclides to become soluble in EA or toluene. Although some hot atoms may be suspended in the organic, they can be removed via washing with dilute aqueous acid. These results can be interpreted to conclude that the radionuclides remaining in the organic solvent after washing are in a location that cannot be accessed by the aqueous solution during vortexing. The only such location known to exist in this system is inside of the fullerene.

3.2.3 Direct Recoil into Esters Towards the end of the project, it occurred to us that perhaps we should try to recoil the daughter radionuclides directly into the fullerene esters. While this would save only a small amount of time, it paves the way for recoil directly into the fullerene acids, which could save several hours, depending on the work-up required to remove exohedral radionuclides. Also, a specific $\mathrm{C}_{60}$ ester (one with six malonic esters, for example) could be used to coat the mesh, resulting in a more homogenous product.

A mixture of $\mathrm{C}_{60}$ Bingel esters (primarily those with 4, 5, and 6 malonic ester groups) was prepared, giving a deep red EA solution. This was then air brushed as before onto a Pt mesh containing $41 \mu \mathrm{Ci}$ of ${ }^{224} \mathrm{Ra}$ and $52 \mu \mathrm{Ci}$ of ${ }^{225} \mathrm{Ac}$ (amongst other radionuclides in the ${ }^{224 / 225} \mathrm{Ra}$ decay chains). The ester-covered mesh was left to sit in air for 15.0 hours, then placed in a scintillation vial and gently vortexed ( $600 \mathrm{rpm})$ in $1.0 \mathrm{~mL}$ EA. The vial and syringe were washed with an additional $0.2 \mathrm{~mL}$ EA, which was combined with the first $\mathrm{mL}$ in a centrifuge tube. Another $0.5 \mathrm{~mL}$ was added to wash, but was basically clear and not 
retained. The EA volume was reduced to $0.7 \mathrm{~mL}$ under flow of $\mathrm{N}_{2}$. The EA solution was then washed as before, with the activities present in each washing fraction shown in Figure 8Figure .

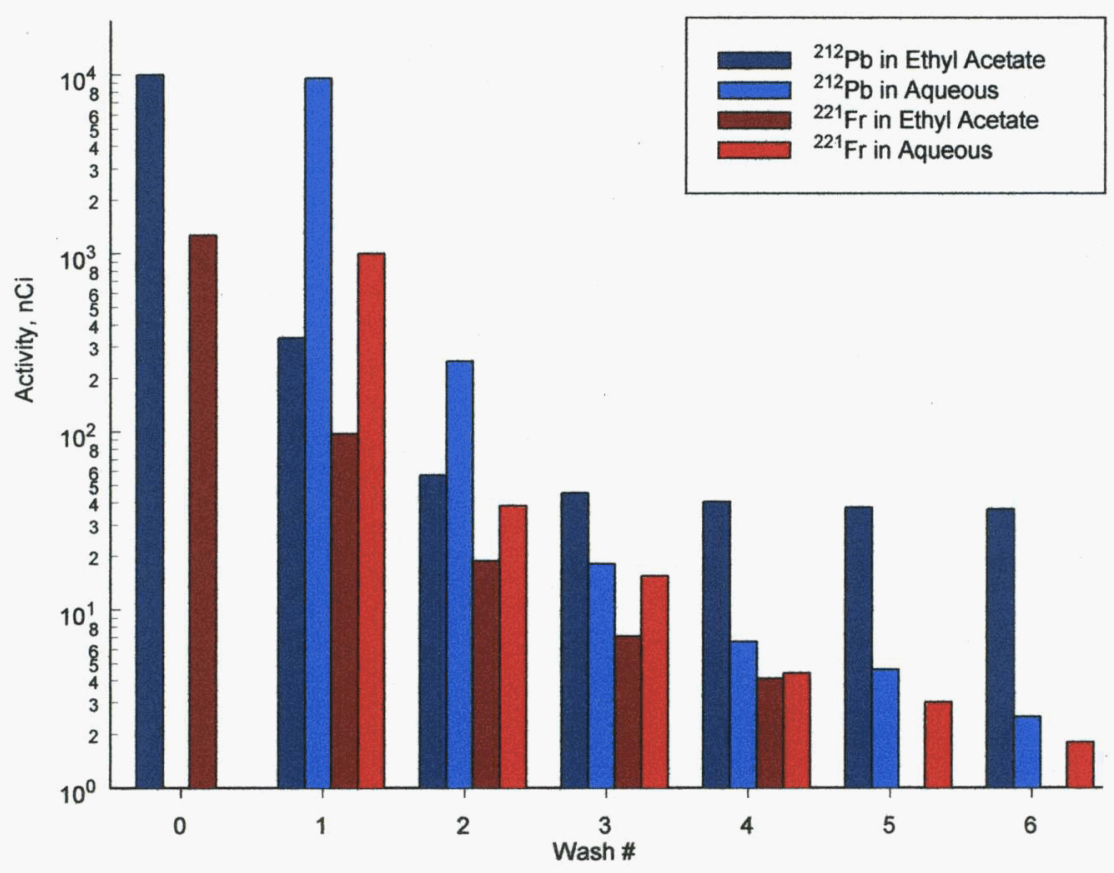

Figure 8: Successive washings of ${ }^{212} \mathrm{~Pb} @ \mathrm{C}_{60}$ malonic ester derivatives in ethyl acetate with dilute nitric acid containing cold lead atoms. Removal of ${ }^{225} \mathrm{Ac}$ atoms not recoiled into the $\mathrm{C}_{60}$ cage is also demonstrated. The average time between washes is six minutes.

Using the same calculations as before, it was found that the encapsulation efficiency for ${ }^{212} \mathrm{~Pb}$ was $0.15 \%$, and that for ${ }^{213} \mathrm{Bi} 0.36 \%$. Although these are at the low end of the range of encapsulation efficiencies, the deviation is not statistically significant. It is interesting to note, however, that it appears to be more difficult to wash out the exohedral radionuclides than for the normal process. Somewhat surprisingly, it therefore appears possible to recoil an atom into a fullerene derivative without greatly disturbing the exohedral derivatization of the fullerene.

\subsubsection{The Fate of the ${ }^{212} \mathrm{Bi}$ From ${ }^{212} \mathrm{~Pb} @ \mathrm{C}_{60}$ Malonic Ester Derivatives Certain reports in the} literature suggest that the $\mathrm{C}_{82}$ fullerene cage could sometimes withstand a beta decay from an endohedral atom (Kikuchi et al., 1994; Sueki et al., 1999), but not all the time (Sueki et al., 1998). However, their criterion for "stability" appeared to be whether the $\mathrm{C}_{82}$ fullerene remained soluble in toluene-like solvents following beta decay. It is now known (Diener and Alford, 1998) that most endohedral metallofullerenes are insoluble, including many isomers of the $\mathrm{C}_{82}$ cage. It is therefore more likely that the beta decays resulting in "instability" merely result in an insoluble endohedral $\mathrm{C}_{82}$. We did not, and still do not, believe that it is possible for the weak recoil energy associated with beta decays to remove an endohedral atom from a fullerene. One of the potential advantages of the fullerene for ${ }^{212} \mathrm{~Pb}$ versus chelators is that the fullerene will retain encapsulation of the daughter ${ }^{212} \mathrm{Bi}$. DOTA loses the ${ }^{212} \mathrm{Bi} 36 \%$ of the time (Mirzadeh et al., 1993). 


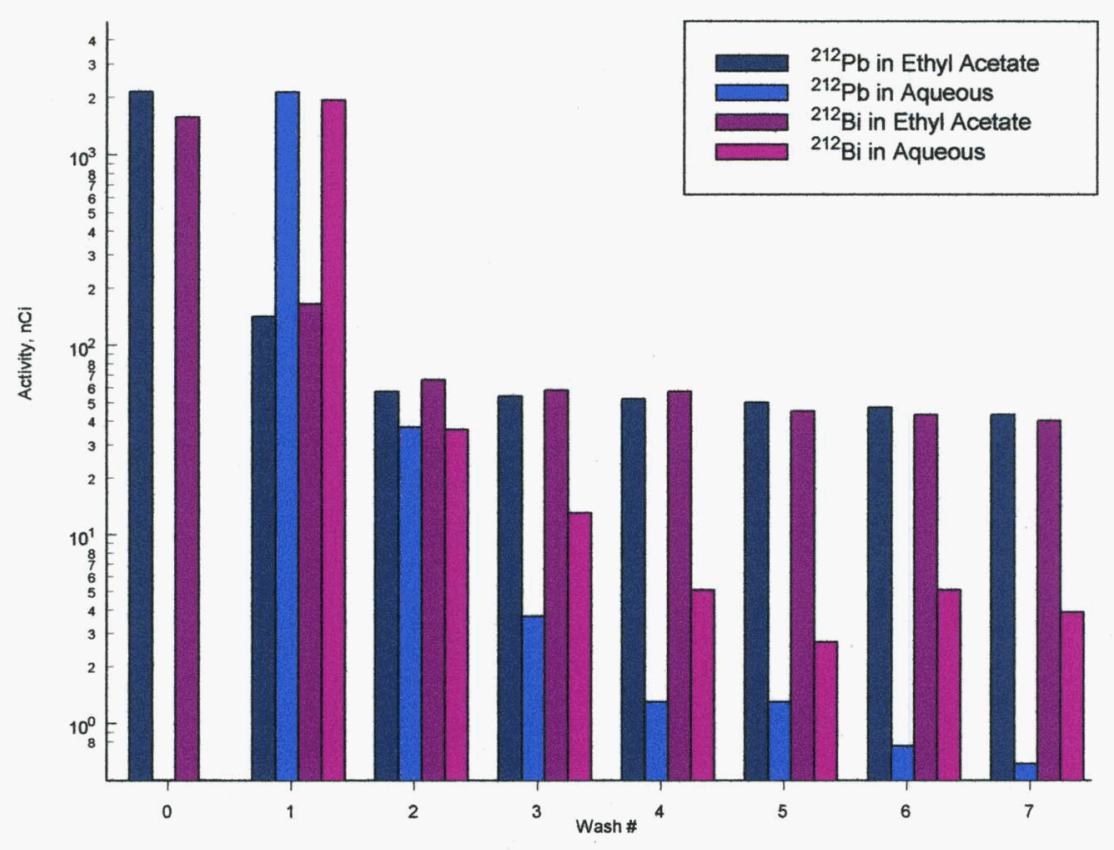

Figure 9: Comparison of ${ }^{212} \mathrm{Bi}$ activity during washing to that of ${ }^{212} \mathrm{~Pb}$.

We were therefore frustrated to consistently find more ${ }^{212} \mathrm{Bi}$ in the aqueous portion following a wash than ${ }^{212} \mathrm{~Pb}$ (Figure 9). So a particular aqueous fraction was counted repeated, and the decay of ${ }^{212} \mathrm{Bi}$ activity (as measured by the $583 \mathrm{eV}$ photon intensity decay) was recorded (Figure 10). It was found that activity can be curve fit by plotting the decay of activity from ${ }^{212} \mathrm{Bi}$ and a little ${ }^{212} \mathrm{~Pb}$ in the solution. From these data it was determined that $36 \%$ of the ${ }^{212} \mathrm{Bi}$ is not retained in the organic.

The most likely explanation for this is that the shakeoff of electrons from the newly formed ${ }^{212} \mathrm{Bi}$ atom sufficiently alters the electron distribution of the $\mathrm{C}_{60}$ so as to degrade the malonic esters responsible for the endohedral fullerene being soluble in the EA. While we cannot prove that the ${ }^{212} \mathrm{Bi}$ is retained inside the fullerene, this seems a far more likely explanation than release of the ${ }^{212} \mathrm{Bi}$ from its fullerene cage.

The implications of this for RIT are hard to predict. It may be that the shakeup of fullerene electrons will not affect the linkage between the radiofullerene and the targeting agent. It may also be that $36 \%$ of the $\alpha$ emitting radionuclides become separated from the targeting agent. What

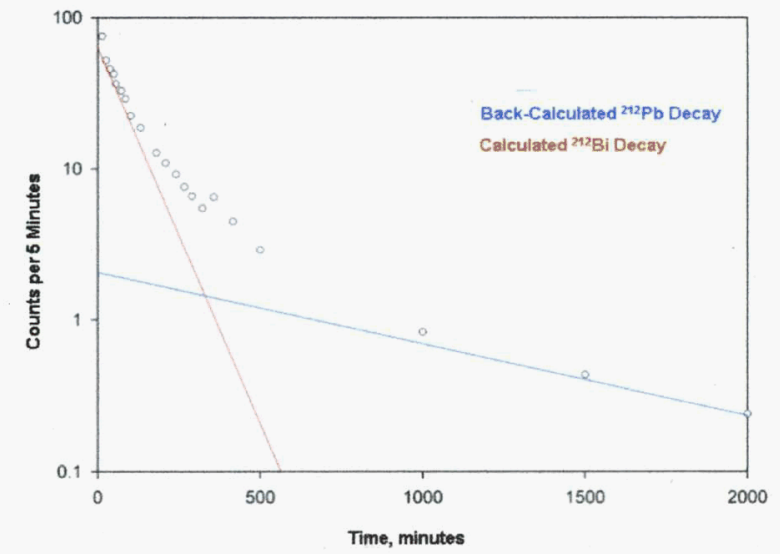

Figure 10: Counting an aqueous wash of $\mathrm{C}_{60}$ esters, showing decay of the ${ }^{212} \mathrm{Bi}$ to secular equilibrium with the ${ }^{212} \mathrm{~Pb}$ in the wash.

remains on the fullerene cage is unclear, except that whatever is left is not sufficient to keep the fullerene soluble in EA. It may be that the degraded fullerene is accumulated in the liver, or it may be that it is expelled. Either way, if an internalizing $\mathrm{Ab}$ is used, the rate of ${ }^{212} \mathrm{Bi} @ \mathrm{C}_{60}$ escape from cells will almost 
certainly be far slower than the rate of free ${ }^{212} \mathrm{Bi}$ escape. A significant portion, if not all of the ${ }^{212} \mathrm{Bi} @ \mathrm{C}_{60}$ may still decay inside of the target cell. Thus, while this is obviously not the most desirable result, it is also not certain to be particularly detrimental either.

3.2.5 Conversion of the Ester to the Acid Having washed away all radionuclides external to the fullerene ester (Section 2.2.1), the ester was now ready to be converted to the water-soluble malonic acid derivative. The basic procedure is given in Figure 11. The EA containing the ester was transferred to a $\sim 10 \mathrm{~mL}$ flask which would serve as the reaction vessel for the hydrolysis. A stream of $\mathrm{N}_{2}$ both purged the flask and evaporated the EA. About $4 \mathrm{~mL}$ of toluene was added to $1-2 \mathrm{mg}$ of $\mathrm{NaH}$ under inert conditions to form a slurry which was then added by syringe to the purged flask. This flask, still under $\mathrm{N}_{2}$, was immersed in a water bath, stirred, and heated to $\sim 80 \mathrm{C}$ for about an hour. It was then removed from the bath, and $\mathrm{MeOH}$ was added immediately but very slowly. The reaction of the $\mathrm{MeOH}$ with the $\mathrm{NaH}$ causes much frothing, even with a tiny drop. Methanol addition was discontinued until the frothing subsided. Suddenly, after the addition of ca. $0.2 \mathrm{~mL}$ of methanol, a red precipitate forms and the toluene turns clear. Methanol addition and stirring can be discontinued at this point.

The toluene was then removed and analyzed for radioactivity, then $\sim 1$ $\mathrm{mL}$ of deionized water was added to the flask, which immediately dissolves the precipitated fullerene acid. The water was transferred to a centrifuge tube, and the solids spun down. Assay of all three portions revealed yields between 55 and $75 \%$ for the ${ }^{212} \mathrm{~Pb}$ fullerenes, with $\sim 7 \%$ left in the toluene and insoluble material.

At this point, the water is extremely basic, $\mathrm{pH} \sim 14$. While this can be neutralized with acid, that leaves a lot of salt in the solution, which proved to be detrimental to the mice (Section 2.3). The process was improved upon by adding $6 \mathrm{M} \mathrm{HCl}$ to the basic water, fully protonating the fullerene acid, and causing it to precipitate. This was then spun down in an mini-ultracentrifuge (the precipitate is very fine), and the acid supernatant syringed off. MES buffer was then added to re-dissolve the fullerene acid. The $\mathrm{pH}$ was then 4 , so a little $1 \mathrm{M} \mathrm{NaOH}$ was added to bring it up to 7 . The precipitation from acid and re-dissolution in buffer proceeded with quantitative yield when the ultracentrifuge was used; but only $75 \%$ of the fullerene acid was recovered with a standard bench top centrifuge $(\sim 3000 \mathrm{rpm})$.

While it may be possible to perform an acid-catalyzed hydrolysis, shorten the heating time with $\mathrm{NaH}$, or otherwise improve the synthesis, in Phase I we were more interested in minimizing losses to the available material. Since the $\mathrm{R} @ \mathrm{C}_{60}$ derivatives are now in water, the aqueous solution is very susceptible to picking up stray radioactive dust from glassware, needles, and other sources in the hood. Once batch became contaminated with ${ }^{225} \mathrm{Ra}$ and daughters, and the contamination could not be removed. The aqueous solution was passed over ion exchange columns containing sulfate groups. First, a coarser 100200 mesh column was used, but that removed less than $30 \%$ of the contamination. Then a $200-400$ mesh column was used, but again, less than $40 \%$ of the contamination was removed. (The contamination removed was assessed by counting both the aqueous solution and the column.) Gratifyingly, less than 5\% of the ${ }^{212} \mathrm{~Pb}$ remained stuck to the column, and perhaps that could have been removed with additional washing. We believe that the carboxylic acid groups on the fullerene are arranged such that they can 
chelate external metal atoms, and that this chelation is strong enough to compete successfully with the sulfate groups on the resin for both +2 and +3 metals!

\subsection{Mouse Biodistributions of $\left.{ }^{212} \mathrm{~Pb} @ \mathrm{C}_{60}\left(\mathrm{C}_{\left[\mathrm{CO}_{2}\right.}^{-}\right]_{2}\right)_{\mathrm{x}}$}

Although the low amounts of radioactivity available resulted in non-optimum methods being employed to obtain three time points for the biodistribution, the results are consistent with each other, and allow certain conclusions to drawn without doubt. The biodistribution study unfortunately turned out to be a synthesis of two different batches of ${ }^{212} \mathrm{~Pb} @ \mathrm{C}_{60}$ malonic acids. We first purposefully attempted a single time point, as a check on our chemical and analytical methods. However, trouble electroplating the ${ }^{224 / 225} \mathrm{Ra}$ (combined with the intrinsically low encapsulation efficiency) at the time we were set to perform the true biodistribution, resulted in less available activity, and scaling back the number of mice in the study. We therefore were forced to rely upon the one hour time point obtained during the initial trial. Since the methods varied slightly, each experiment is described separately below, and then the data are collated in the third subsection.

3.3.1 One Hour Study As the carboxylic acid derivatives of $\operatorname{Ln} @ \mathrm{C}_{60}(\mathrm{Ln}=$ lanthanide $)$ are apparently water-soluble down to $\mathrm{pH} \sim 5$ (determined from cold work at TDA), we assumed the same for ${ }^{212} \mathrm{~Pb} @ \mathrm{C}_{60}$. After adjusting the $\mathrm{pH}$ of the MES-buffered aqueous solutions containing the ${ }^{212} \mathrm{~Pb} @ \mathrm{C}_{60}$ carboxylate to $6-7$, we were ready to inject the mice.

The first time, we thought we would do a trial study using three mice and sacrificing at one hour, to test the procedure, the preparation of the fullerene acid, and the data collection mechanism. This first time, we dissolved the fullerene acid precipitate from methanol directly into MES, and adjusted the $\mathrm{pH}$ down to $\sim 7$. Adjusting the $\mathrm{pH}$ required $285 \mathrm{ul}$ of $2 \mathrm{M} \mathrm{HCl}$, followed by $20 \mathrm{ul}$ of $8 \mathrm{~m} \mathrm{HCl}$. We did not re-precipitate the fullerene acid. There were then $130 \mathrm{nCi}$ of ${ }^{212} \mathrm{~Pb} @ \mathrm{C}_{60}$ acids in $750 \mathrm{ul}$ of MES-buffered aqueous solution. Two 10ul aliquots were removed as standards for the gamma ray spectroscopy counting. For this test run, we had three female mice with severe combined immunodeficiencies (SCID) ready.

$200 \mu \mathrm{L}$ of the solution were administered by tail vein injection to the first mouse, which promptly expired following injection. Dr. Kennel believed (and believed prior to injection, actually) that the salt concentration of the fullerene acid solution was too high for the mouse. Therefore, the fullerene solution was diluted with an equal part of deionized water, and two injections of $200 \mathrm{ul}$ total volume each (where each injection contained $100 \mathrm{ul}$ of the fullerene solution and $100 \mathrm{ul}$ of deionized water) were made two minutes apart for the second mouse. Despite hunching and some impaired mobility, the mouse lived. The third SCID was also injected in this manner, but the injections were made more slowly, the mouse exhibited less hunching. To obtain a third data point, the remaining $125 \mathrm{ul}$ of fullerene solution (diluted with $125 \mu \mathrm{L} \mathrm{DI} \mathrm{H}_{2} \mathrm{O}$ ) was administered in a single injection to a BALB/C mouse.

The mice were sacrificed one hour after injection by cervical dislocation. The following tissues were collected from each mouse and placed in previously tared plastic scintillation vials: skin, leg muscle, femur and sternum (referred to as "bone" henceforth), stomach and intestines (which were emptied first), ovaries and uterus, liver, spleen, kidneys, heart, and lungs. Blood, including any available clots, was also collected.

The vials were then counted in a Wallac Wizard. The Wallac is far more sensitive than the spectrometer discussed on page 4, but lacks the resolution. It was preset to collect all photons emitted from the sample over two $\gamma$-ray energy ranges; the first corresponding the $238 \mathrm{keV} \gamma$-ray from ${ }^{212} \mathrm{~Pb}$, and the second to the 510 and $583 \mathrm{keV} \gamma$-rays from ${ }^{208} \mathrm{Tl}$ used to assess ${ }^{212} \mathrm{Bi}$. As there were no other radioisotopes present in the sample (determined by counting a few tissues on the spectrometer), there were no interferences. Tissues were counted for 10 minutes each. The $10 \mu \mathrm{L}$ standards were also counted, as were blank vials to 
provide a background. The Wallac provides data in the form of counts per sample per photon energy range, along with the time that the counting was concluded.

Subtracting the average counts associated with the blank vials from each of the tissue counts gave a record of counts per 10 minutes per tissue for each isotope. Using the time stamp and the half life of ${ }^{212} \mathrm{~Pb}$ allowed the calculation of the ${ }^{212} \mathrm{~Pb}$ counts at the time of sacrifice for each tissue of each mouse. The total injected dose was calculated from the 10 ul aliquot counts, and the activity calculated from the counting the aliquots in the spectrometer. From this, it was determined that the first two mice received $28 \mathrm{nCi}$ of ${ }^{212} \mathrm{~Pb}$, and the third mouse received $18 \mathrm{nCi}$. The vials were reweighed the following day to determine the weights of each the tissues collected. Since it took so long to count the tissues, ${ }^{212} \mathrm{Bi}$ was either back in equilibrium with the ${ }^{212} \mathrm{~Pb}$, or had decayed away. Deviations from equilibrium were well within the uncertainty in the ${ }^{212} \mathrm{~Pb}$ activity. The data was assembled into the biodistribution graph shown in Figure 12Figure . Considering the variation in method of administration, the data are in reasonable agreement from mouse to mouse.

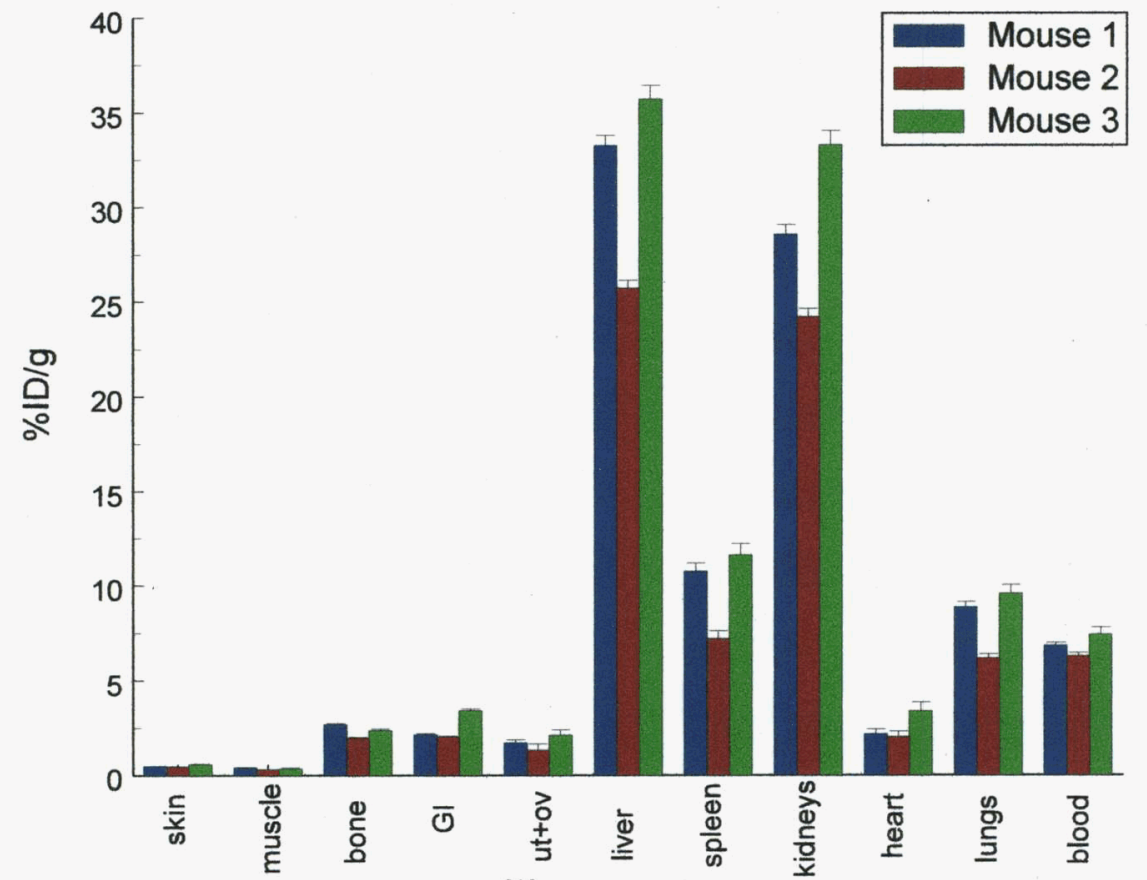

Figure 12: One hour biodistribution of ${ }^{212} \mathrm{~Pb} @ \mathrm{C}_{60}\left[\mathrm{C}\left(\mathrm{COO}^{-}\right)_{2}\right]_{\mathrm{x}}$, including standard error.

See text for details.

\subsubsection{Three Hour and Eight Hour Biodistributions}

ready to attempt the biodistibution study, we knew what the encapsulation efficiency was, and, while we had experienced some problems with electroplating $\mathrm{Ra}$, they had not been severe. Unfortunately, during the days leading up to the biodistribution, it seemed that only $\sim 10 \%$ of the $\mathrm{Ra}$ in solution could be electroplated onto the mesh. (We had previously achieved $30-50 \%$ ). It was decided to use three meshes, esterify and wash the $\mathrm{C}_{60}$ coating each mesh, and combine the esters prior to acidification. As described in Section 2.2.5, we avoided the problem of excess salt (and base) by re-precipitating the fullerene acid following the initial aqueous dissolution with $6 \mathrm{M} \mathrm{HCl}$, followed by ultracentrifugation, removal of the supernatant, and redissolution in MES (followed by a minor $\mathrm{pH}$ adjustment). However, there were only $55 \mathrm{nCi}$ of ${ }^{212} \mathrm{~Pb} @ \mathrm{C}_{60}\left[\mathrm{C}\left(\mathrm{COO}^{-}\right)_{2}\right]_{\mathrm{x}}$ left for the mice. Thus, we were forced to back off some of the planned time points in order to have enough activity to do six mice well. 
Six BALB/C mice received 200 ul of fullerene acids in MES by a single tail vein injection, 9 nCi per mouse. The mice did not exhibit an of the hunching or discomfort previously seen, and appeared unaffected by the injection. Three mice were sacrificed at 3 hours post-injection, and the remaining three at 8 hours post-injection. The same tissues were collected as before, with the addition of collecting the urine expelled at the time of death. The activities and tissue weights were assessed using the same procedures as for the 1 hour biodistribution. However, certain tissues from the $8 \mathrm{~h}$ mice were recounted the following morning for 30 minutes each on the Wallac to improve the signal to noise ratio. The tissues selected were those perceived to be the more important ones, namely bone, spleen, kidneys, liver, blood, urine, and stomach. The individual mouse biodistributions for $3 \mathrm{~h}$ and $8 \mathrm{~h}$ are shown in Figure 13 Figure .
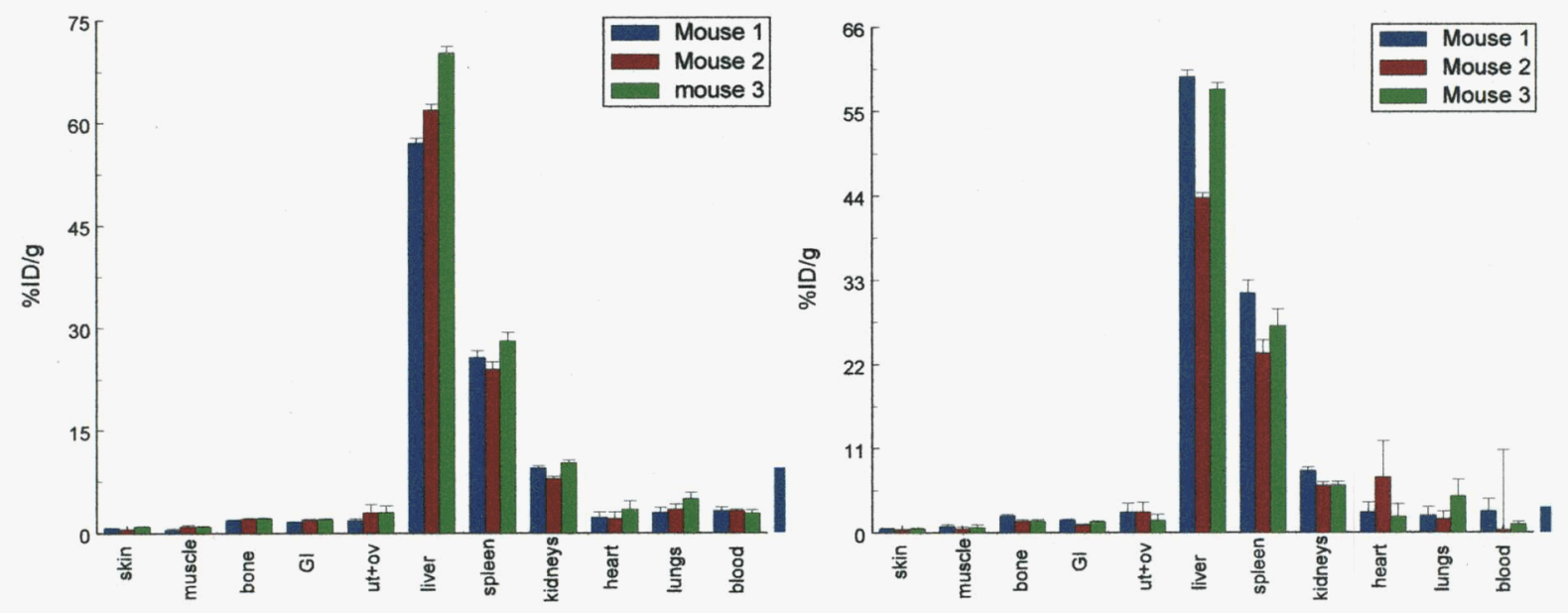

Figure 13: Three hour (left) and eight hour (right) biodistributions of ${ }^{212} \mathrm{~Pb} @ \mathrm{C}_{60}\left[\mathrm{C}\left(\mathrm{COO}^{-}\right)_{2}\right]_{\mathrm{x}}$.

\subsubsection{Average Biodistribution; Comparison to Previous Studies To provide the usual} biodistribution graph, the $\% \mathrm{ID} / \mathrm{g}$ for each tissue were averaged for the three mice from each time point. The combined "average" biodistribution graph is shown in Figure 14Figure . Despite the unusual preparation method, some conclusions can still be drawn with confidence from data.

Like other fullerene derivatives so far investigated, the malonic acids derivative also seems to accumulate primarily in the liver. By normalizing the counts from the liver to the $10 \mu \mathrm{L}$ aliquots, it seems that about $50 \%$ of the injected dose accumulates in the liver. We had hoped to avoid this with the carboxyfullerene, and achieve rapid clearance, but clearly that did not happen. Data recently presented at the Material Research Society Meeting on Dec 2, 2003 from Prof. Wilson's group at Rice used dynamic light scattering (DLS) to assess the aggregate size of $\mathrm{Gd} @ \mathrm{C}_{60}$ carboxyfullerenes prepared at TDA Research, and found that the fullerenes aggregated significantly, even at $\mathrm{pH}$ 7. This may explain the accumulation in the liver. 


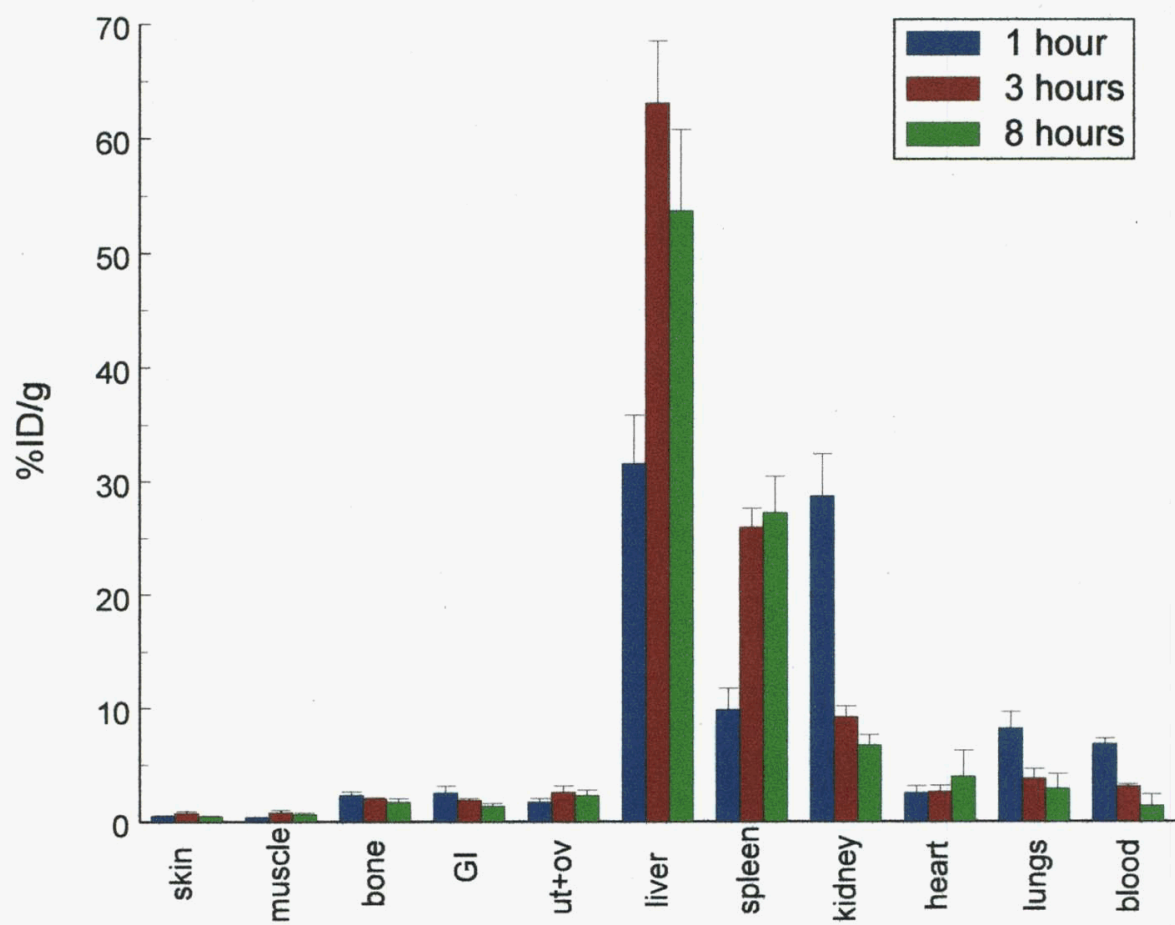

Figure 14: Average biodistribution of ${ }^{212} \mathrm{~Pb} @ \mathrm{C}_{60}\left[\mathrm{C}\left(\mathrm{COO}^{-}\right)_{2}\right]_{\mathrm{x}}$ using the data presented in the previous two subsections. "Error bars" show standard deviation.

The extra residence time in the liver was however useful since it demonstrated that the ${ }^{212} \mathrm{~Pb}$ is not released to the bone, the target tissue for lead. The slight accumulation in the bone $(2.3$ at $1 \mathrm{~h}, 1.8$ at $\mathrm{h}, 1.5$ at $8 \mathrm{~h} \% \mathrm{ID} / \mathrm{g}$ ) both decreases with time, and compares favorably to the previous DOTA-Ab biodistribution study (Ruble et al., 1996, found 3.2-7.8 \% ID/g depending on the targeting agent). Thus, whatever is happening to the fullerene in the liver (if anything) does not release the endohedral ${ }^{212} \mathrm{~Pb}$. This is a crucial point, since DOTA-chelated ${ }^{212} \mathrm{~Pb}$ tends to result in severe myelotoxicity, presumably from freed ${ }^{212} \mathrm{~Pb}$ accumulation in the bone. A targeted ${ }^{212} \mathrm{~Pb}$ fullerene should have even less accumulation in the bone, as the targeting agent will direct the fullerene to a specific tissue.

Amongst other tissues, clearance from the blood and kidneys is demonstrated, indicating that the free ${ }^{212} \mathrm{~Pb} @ \mathrm{C}_{60}\left[\mathrm{C}\left(\mathrm{COO}^{-}\right)_{2}\right]_{\mathrm{x}}$ clears from circulation on a time scale commensurate with the half life of ${ }^{212} \mathrm{~Pb}$. Significant accumulation in the spleen is also noted, again, a possible result of aggregation. Doses to other tissues are low; the free carboxyfullerene does not target any of the other tissues sampled.

The chemistry developed on this project is clearly a significant improvement over the only previous endohedral radiofullerene biodistribution study (Cagle et al., 1999). The authors of that study prepared a polyhyroxylated ${ }^{166} \mathrm{Ho} @ \mathrm{C}_{82}$ by neutron activation of the ${ }^{166} \mathrm{Ho}$. The polyhydroxylated radiofullerene obtained $8 \% \mathrm{ID} / \mathrm{g}$ bone, and accumulated over time. They also observed very high doses of the fullerene in the liver, which cleared slowly, and similarly low doses with significant clearance from the other tissues assessed in this study. From our preliminary study, it appears that the malonic acid derivatization is superior to polyhydroxylation for radiofullerenes.

The most important part of the biodistribution study is that the free ${ }^{212} \mathrm{~Pb} @ \mathrm{C}_{60}$ derivative does not accumulate in the bone. Therefore any catabolism, or any other biological process that competes for $\mathrm{Pb}$, will not result in uptake of ${ }^{212} \mathrm{~Pb}$ in bone from the endohedral fullerene ${ }^{212} \mathrm{~Pb} @ \mathrm{C}_{60}$. This use of the fullerene is so far the only known method to prevent ${ }^{212} \mathrm{~Pb}$ uptake in bone, and will presumably mitigate 
the myelotoxicity that plagued earlier studies (Ruble et al., 1996; Horak et al, 1997) and has so far prevented the further development of ${ }^{212} \mathrm{~Pb}$ radiopharmaceuticals.

\subsection{Summary of ${ }^{212} \mathrm{~Pb}$ Studies}

The primary achievement of this part of the project was that incontrovertible evidence was obtained for the production of ${ }^{212} \mathrm{~Pb} @ \mathrm{C}_{60}$ via recoil implantation following $\alpha$-decays in the ${ }^{224} \mathrm{Ra}$ chain. We also established a rapid protocol for the chemical derivatization of the hot endohedral fullerene, leading to a robust, non-aggregating water-soluble radiofullerene derivative that is easily separated from both hot and cold unencapsulated nuclides. Although the fullerene did not show improvement vs. chelators at containment of the ${ }^{212} \mathrm{Bi}$ daughter, the fullerene does unequivocally prevent leakage of the bone-targeting ${ }^{212} \mathrm{~Pb}$. This is the key requirement for further development of ${ }^{212} \mathrm{~Pb}$ for RIT that has not yet been surmounted by chelation techniques. In summary, we have demonstrated the promise of the $\alpha$-recoil implantation technique for producing radiofullerenes suitable for pharmaceutical development, met only readily surmountable hurdles, and remain enthusiastic about the continued development of the technique.

\section{Implantation of ${ }^{177} \mathrm{Lu}$ by Recoil Following Neutron Capture}

The third method pursued in this project uses the energy from the recoil following neutron absorption to implant the activatved atom into the fullerene. Depending upon the isotope, the energy of the neutron capture reaction is usually between 5 and $7 \mathrm{MeV}$ and is radiated as $\gamma$-rays. Upon emission of a $\gamma$-ray, the nucleus recoils with energy $\sim 120 \mathrm{eV}$ (for lanthanides with an average mass of $\sim 130 \mathrm{amu}$ ). As the nucleus recoils through the fullerene lattice it can eventually break open a fullerene and become trapped inside. The large number of vibrational modes in the fullerene rapidly redistributes and dissipates the collision energy, thus allowing the ruptured bonds to reform. This techniques has been used for ${ }^{7} \mathrm{Be}$ (Ohtsuki et al., 1994), ${ }^{41} \mathrm{Ar}$ (Gadd et al. 1997,1998; Braun and Rausch 1995), various $\mathrm{Xe}$ and $\mathrm{Kr}$ isotopes (Gadd et al. 1997), ${ }^{140} \mathrm{La}$ (Rausch and Braun, 2001), and ${ }^{69} \mathrm{Ge},{ }^{72} \mathrm{As},{ }^{75} \mathrm{Se},{ }^{120,}{ }^{122} \mathrm{Sb}$, and ${ }^{121} \mathrm{Te}$, (Ohtsuki et al. 2000, 2001, and 2002), although it is difficult to determine whether the p-electron atoms are inside of the fullerene or have become part of the fullerene cage. Of particular interest, the experiments performed by Braun and Rausch (1998) appeared to demonstrate that it is possible to produce significant yields of radioactive metallofullerenes by irradiating mechanically homogenized mixtures of $\mathrm{C}_{60}$ and metal oxides of $\mathrm{Cu}, \mathrm{La}, \mathrm{Pr}, \mathrm{Ho}$, and $\mathrm{Zn}$. By dissolving the product in aniline and subsequently extracting the aniline with aqueous acid, the authors conclude that a significant fraction of the activated metals become trapped inside the $\mathrm{C}_{60}$ during the activation process. The production yields calculated by the authors are ${ }^{63} \mathrm{Cu} \rightarrow$ ${ }^{64} \mathrm{Cu} @ \mathrm{C}_{60}(9.0 \%),{ }^{139} \mathrm{La} \rightarrow{ }^{140} \mathrm{La} @ \mathrm{C}_{60}(14.5 \%),{ }^{140} \mathrm{Pr} \rightarrow{ }^{141} \mathrm{Pr} @ \mathrm{C}_{60}(31.1 \%),{ }^{165} \mathrm{Ho} \rightarrow{ }^{166} \mathrm{Ho} @ \mathrm{C}_{60}(3.6 \%)$, and ${ }^{64+68} \mathrm{Zn} \rightarrow{ }^{65+69 m} \mathrm{Zn} @ \mathrm{C}_{60}(9.1 \%)$. These results are in general agreement with our previous results (Thrash et al, 1999), which examined the escape of activated ${ }^{166} \mathrm{Ho}$ from inside pre-synthesized ${ }^{165} \mathrm{Ho} @ \mathrm{C}_{82}$. However, the results from this part of the project raised serious questions concerning Braun and Rausch's procedures and results.

We selected ${ }^{177} \mathrm{Lu}$ for several reasons. The advantages and disadvantages of ${ }^{177} \mathrm{Lu}$ use in RIT were discussed in the original report of its use in a radiologic immunoconjugate (Schlom et al., 1991). Its long 6.7-day half-life provides ample time to develop the requisite purification and derivatization procedures without substantial loss of activity or large drop in specific activity. The ability of the fullerene to encapsulate the radionuclide with zero in-vivo loss is always important, but is even more crucial for a long half-life radionuclide. Current chelates such as DOTA perform very well, but as the half-life increases, so does the chances that transchelation will eventually release the radionuclide. Free lanthanides such as ${ }^{177} \mathrm{Lu},{ }^{166} \mathrm{Ho}$, and ${ }^{90} \mathrm{Y}$ strongly target bone where they irradiate the bone marrow, and in many cases this is the dose-limiting factor. The stringent requirements for chelation were considered to be the primary disadvantage for ${ }^{177} \mathrm{Lu}$ development in the Schlom publication, and the fullerene is the best choice to 

barns while $\sigma^{0}{ }_{176}=2090$ barns, Mughabghab et al. 1981). Because of this large difference and the high cross section of ${ }^{176} \mathrm{Lu}$, activating a mixture of naturally occurring $\mathrm{Lu}$ and $\mathrm{C}_{60}$ should provide encapsulated ${ }^{177} \mathrm{Lu} @ \mathrm{C}_{60}$ with both high activity and high specific activity. While the primary pathway for formation of ${ }^{177} \mathrm{Lu} @ \mathrm{C}_{60}$ is activation of ${ }^{176} \mathrm{Lu}$, our model includes

all of the known reactions. To estimate the ${ }^{177} \mathrm{Lu}-\mathrm{C}_{60}$ trapping efficiency, we used the lowest encapsulation value reported by Braun and Rausch (3\%). The efficiency of keeping the previously encapsulated ${ }^{176} \mathrm{Lu}$ in the fullerene during activation is estimated at $25 \%$ based Thrash et al. (1999). Survival of the beta decay of ${ }^{177} \mathrm{Lu}$ to ${ }^{177} \mathrm{Hf}$ is assumed to $100 \%$ as reported Kikuchi et al. (1994).

The reaction equations were formulated into series of simultaneous ODE's that were subsequently solved by numerical integration to yield the concentration of each of the species in the model as a function of the irradiation time. For our test model, we assumed that $100 \mathrm{mg}$ of $\mathrm{C}_{60}\left(1.4 \times 10^{-4} \mathrm{~mol}\right)$ was mixed uniformly with a six to one ratio of $L u$ to $C_{60}$ (a reasonable value for $C_{60}$ intercalates), and that this sample ( $600 \mathrm{mg}$ of natural $\mathrm{Lu}_{2} \mathrm{O}_{3}, 6: 1$ ratio of $\mathrm{Lu}$ to $\mathrm{C}_{60}$ ) would be irradiated at the HFIR's pneumatic tube one facility, which has a neutron flux of $2.3 \times 10^{14} \mathrm{n} \mathrm{sec}^{-1} \mathrm{~cm}^{-2}$. The estimated yield and specific activity of ${ }^{177} \mathrm{Lu} @ \mathrm{C}_{60}$ predicted by the model are shown in Figure 15Figure. It is readily apparent that ${ }^{177} \mathrm{Lu} @ \mathrm{C}_{60}$ with an activity of almost $1 \mathrm{Ci}$ can be easily obtained even for this relatively small sample that is irradiated in a low flux position of the HFIR. The specific activity, expressed as the percent purity of the ${ }^{177} \mathrm{Lu} @ \mathrm{C}_{60}$, can vary between 70 and $85 \%$ depending upon the desired activity, a value that is certainly acceptable for radiotherapy. We believe that this calculation illustrates our point that high activity and specific activities
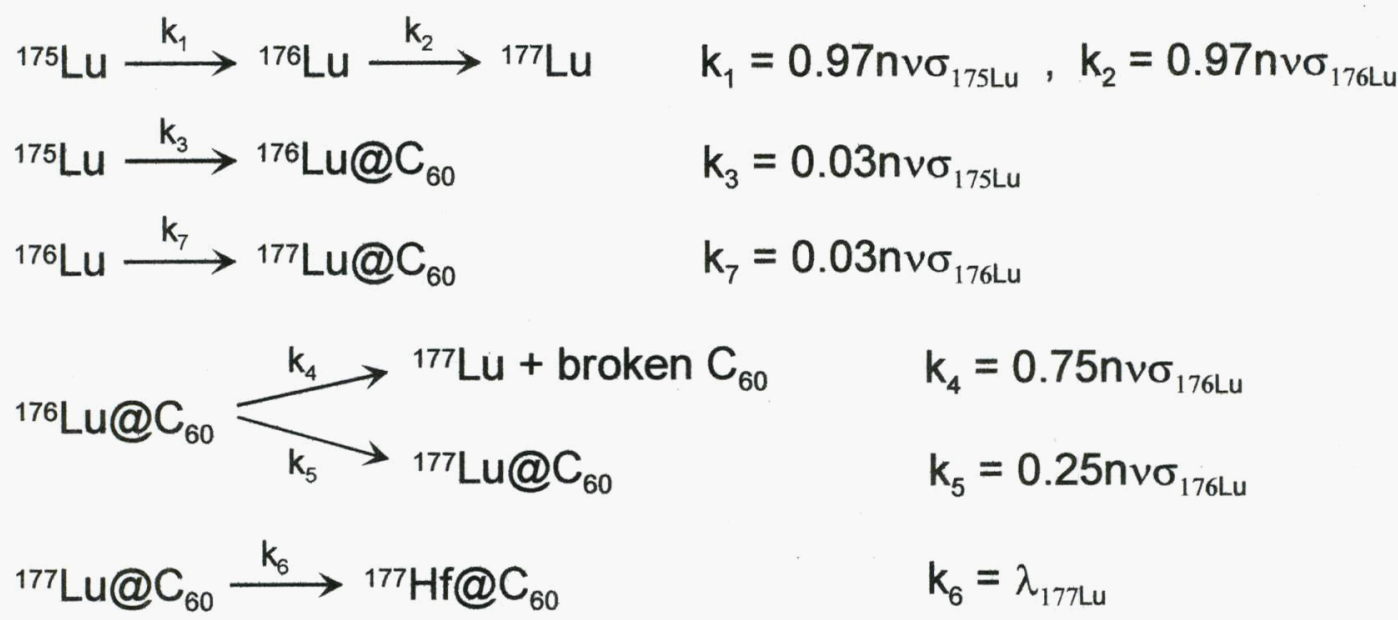

Figure 15. Kinetic model for the production of ${ }^{177} \mathrm{Lu} @ \mathrm{C}_{60}$ by recoil implosion in a nuclear reactor. Where $\mathrm{n}$ is the number of Lu nuclide in the target, and $v$ is the flux density of thermal neutron

can be readily attained by the recoil method (assuming the data in Braun and Rausch, 1998, is accurate). If commercial production was required it would be relatively easy to irradiate a sample 100 times larger than this at the high flux position of the HFIR, which has a flux of $2 \times 10^{15} \mathrm{n} \mathrm{sec}^{-1} \mathrm{~cm}^{-2}$. 


\subsection{Neutron Activation to ${ }^{177} \mathrm{Lu}$ in Mixtures of Lutetium Oxide and $\mathrm{C}_{60}$}

This section begins with a brief description of the HFIR, and the techniques employed there to produce ${ }^{177} \mathrm{Lu}$ in our samples. It then presents results from activation of the $\mathrm{Lu}_{2} \mathrm{O}_{3}-\mathrm{C}_{60}$ mixtures. Since Braun and Rausch (1998) apparently achieved excellent results from mixtures of metal oxides and $\mathrm{C}_{60}$, we felt that our investigations would use the same technique.

\subsubsection{Neutrons From the HFIR}

The HFIR has a variety of different facilities, at different fluxes and fast/epithermal/thermal neutron content. Since we are solely interested in thermal neutrons, and low fluxes are sufficient for the proof-ofconcept experiments undertaken in Phase I, we used the lowest flux positions on the HFIR, the pneumatic tube facilities of the neutron activation laboratory. Two pneumatic tube facilities, designated PT-1 and PT-2, are available in the HFIR. These facilities, operated by the ORNL Analytical Chemistry Division, consist of flight tubes, air supply and exhaust lines, loading stations at which sample containers (rabbits) are introduced into the flight tubes, and irradiation stations to which the rabbits move to be irradiated (see

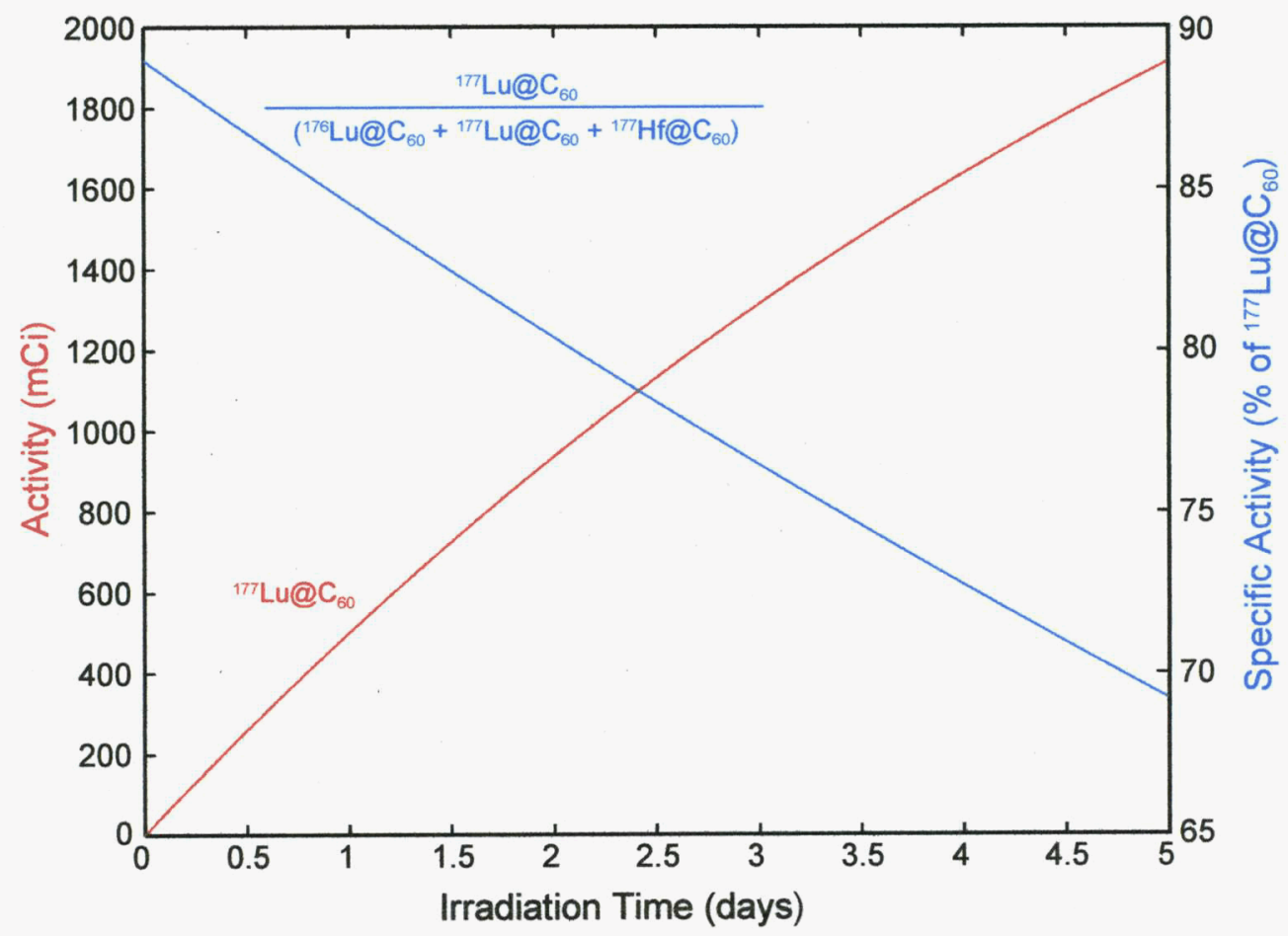

Figure 16. Integrated rate equations yielding the total activity of ${ }^{177} \mathrm{Lu} @ \mathrm{C}_{60}$ and the specific activity of ${ }^{177} \mathrm{Lu} @ \mathrm{C}_{60}$ as a function of the irradiation time. The specific activity is calculated as the percent of ${ }^{177} \mathrm{Lu} @ \mathrm{C}_{60}$ relative to all endohedral $\mathrm{C}_{60}$ species produced.

Figure 17Error! Reference source not found.). The inner diameter of the flight tubes is $0.62 \mathrm{in.}$ (15.88 $\mathrm{mm})$, and the outer diameter of the rabbit is $0.56 \mathrm{in}$. $(14.48 \mathrm{~mm})$. Both flight tubes accept the same rabbits, which have an internal volume of about $1 \mathrm{~cm}^{3}$. Both systems operate with air entering both ends of the flight tube. Capsules are inserted into the reactor and returned to shielded loading stations in the laboratory. The capsules stop on air columns, which permit them to be made of graphite as well as plastic. The capsules can be stopped at decay stations in the pool if they are temporarily too radioactive to return to the laboratory. 
PT-2 is located in a slant engineering facility that intersects the outer edge of the permanent beryllium reflector. (The $\mathrm{Be}$ reflector forms a shield around the core.) This places the irradiation station approximately $12.5 \mathrm{in} .(32 \mathrm{~cm})$ from the fuel element. The thermal flux for the facility is about $5.9 \times 10^{13}$ neutrons $/\left(\mathrm{cm}^{2} \cdot \mathrm{s}\right)$. The thermal/epithermal ratio is approximately 200 , minimizing damage to the fullerene shell.

Unfortunately, the HFIR was shut down for administrattive reasons for the bulk of this project. While the HFIR had previously operated from 1966 until 1986 without shutdown (except to change the fuel),

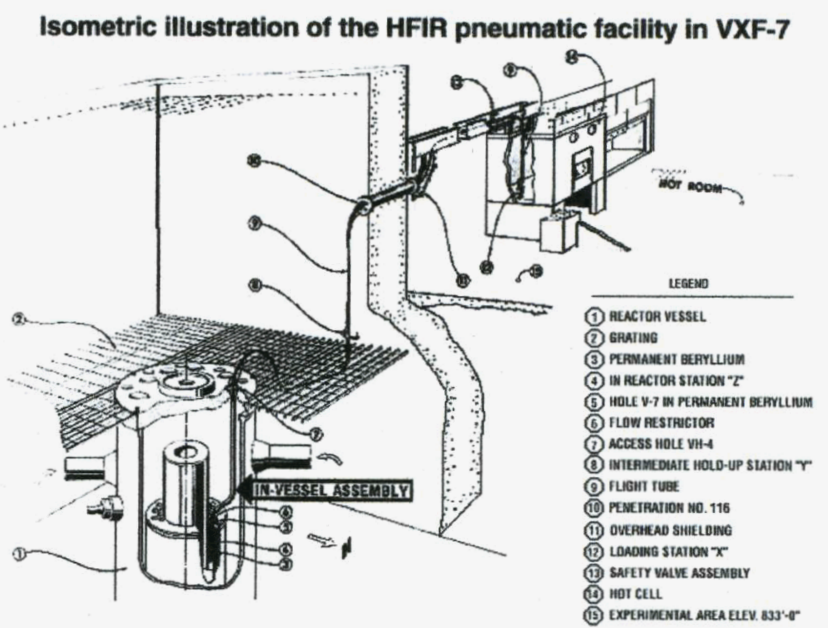

Figure 17: PT-1 of the HFIR; PT-2 was used to neutron activate the samples, and they are similarly designed and located. multiple administrative shutdowns since then have occurred, and this project had the unfortunate timing of nearly coinciding with one the longest. Once the system was restarted, however, the neutron activation team was able to irradiate and return samples of varied chemical composition in a prompt and efficient manner. (It is likely that our samples received something of a priority status during this time, as we made the project's time constraints known to them.)

The implications of the extended downtime of the HFIR for this project were that we had to irradiate many samples more or less at once, often without knowing the results of the previous irradiation. This is a relatively inefficient experimental method, compared to a sequential plan of experimentation. In analyzing the data, it became clear that some opportunities were not capitalized on. Nevertheless, we will show in the following subsections that sufficient results were obtained to validate the central concepts of the project, namely that recoil implantation is an attractive method for production of medicinally useful radiofullerenes.

\subsubsection{Irradiation of Natural $\mathrm{Lu}_{2} \mathrm{O}_{3}-\mathrm{C}_{60}$ Mixtures}

The procedure of Braun and Rausch (1998) served as a starting point for our investigations, as they claimed $>3 \%$ of the activated metal became implanted inside of $\mathrm{C}_{60}$ during their irradiations. They mechanically mixed naturally occurring metal oxide powders with $\mathrm{C}_{60}$ and subjected the mixture to a neutron flux of $1.95 \times 10^{13} \mathrm{n} / \mathrm{cm}^{2} / \mathrm{s}$ for $12 \mathrm{~h}$. At TDA, $150 \mathrm{mg}$ of each $\mathrm{C}_{60}$ and $\mathrm{Lu}_{2} \mathrm{O}_{3}$ were placed in a plastic vial $1 / 2$ " diameter by 1 " tall. About 7 alumina balls $1 / 4$ " in diameter (mini grinding media) were added to the container. The container was then vibrated at high speed for 30 seconds, producing a uniformly brown, fine powder. Without the mini-media, the black $\mathrm{C}_{60}$ and white oxide grains did not mix well, resulting in a salt-and-pepper like powder.

Three samples were created from the brown powder, with weights $1.5 \mathrm{mg}, 2.0 \mathrm{mg}$, and $3.0 \mathrm{mg}$. The samples were weighed out into lidded polyethylene containers, and were all placed in a rabbit. The rabbit was irradiated for $20 \mathrm{~min}$ in PT-2 with a flux of $5^{*} 10^{13} \mathrm{n} / \mathrm{cm}^{2} / \mathrm{s}$. Following irradiation, the samples were allowed to cool for several days. 
Each of the three samples and containers was removed from the rabbit, placed in separate vials, and counted individually in a gamma gay spectrometer. A typical gamma ray spectrum is shown in Figure 18Figure. The two $\gamma$-rays from ${ }^{177} \mathrm{Lu}$ are clear and distinct, without contamination. Although the ordinate is given normalized in arbitrary units in the figure, the data is taken as counts with a resolution of $0.5 \mathrm{keV}$ per channel. Since the gamma ray energies are actually a few channels wide, the software calculates an area under each peak. The activity of the samples is then calculated by dividing the area of each of the peaks by the counting time, the detector efficiency as a function of the shelf and the gamma ray (previously determined by calibrated standard), and the probability of the gamma ray occurring ( 0.066 for the $112.95 \mathrm{keV}$ photon, 0.110 for the $208.36 \mathrm{keV}$ photon) to give activity in decays per second (dps). Where necessary, the dps values for the two photons were averaged to give a single value for the ${ }^{177} \mathrm{Lu}$ content of the sample. Since dps is a somewhat cumbersome unit, it was divided by $3.7 * 10^{4}$ to give a value in microcuries $(\mu \mathrm{Ci})$.

The initial activity of each of the samples was calculated from $A_{0}=A / \exp (-\lambda t)$, with $A$ representing the activity at time $\mathrm{t}$, and $\lambda$ the decay constant $(=$ $.693 /$ half-life). The initial activity per $\mathrm{mg}$ of $\mathrm{Lu}_{2} \mathrm{O}_{3}$ was then obtained for each of the samples. Thus it was determined that 433,457 , and $459 \mu \mathrm{Ci}{ }^{177} \mathrm{Lu} \min$ in PT-2.

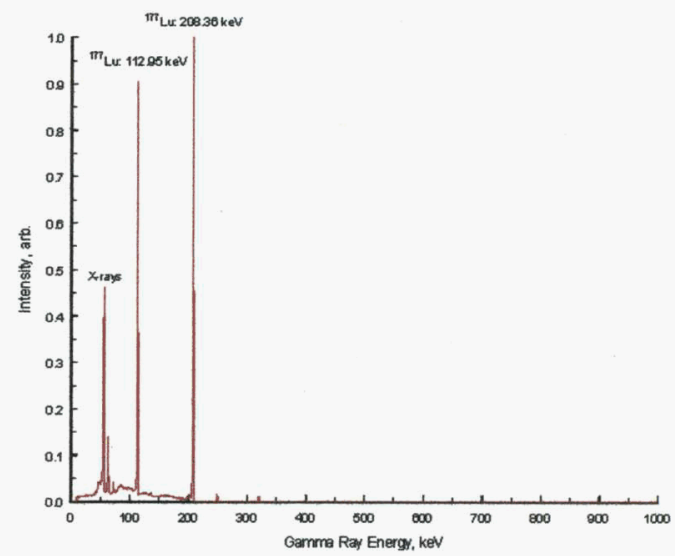

were obtained per $\mathrm{mg}$ of natural lutetium oxide (average $=450$ ) for 20 minutes of irradiation in PT-2 for the $3.0 \mathrm{mg}, 2.0 \mathrm{mg}$, and $1.5 \mathrm{mg}$ samples respectively.

\subsubsection{Activation of ${ }^{176} \mathrm{Lu}_{2} \mathrm{O}_{3}-\mathrm{C}_{60}$ Mixtures}

We also prepared and irradiated mixtures of $70 \%$ enriched ${ }^{176} \mathrm{Lu}_{2} \mathrm{O}_{3}$ and $\mathrm{C}_{60}$. ${ }^{176} \mathrm{Lu}$ is quite expensive $(\$ 172 / \mathrm{mg}$ ) and only a few $\mathrm{mg}$ were readily available at ORNL. Mini-ball milling could not be used to prepare samples, as either a great percentage of the ${ }^{176} \mathrm{Lu}$ would end up stuck to the grinding media, or the ${ }^{176} \mathrm{Lu}$ would be so dilute in the fullerene so as to be disadvantageous during work-up. Therefore, a method was devised, whereupon the ${ }^{176} \mathrm{Lu}$ oxide was sonicated into a known volume of acetone, which was subsequently divided into two portions. One $\mathrm{mg}$ of $\mathrm{C}_{60}$ was added to both portions, and they were placed into polyethylene containers suitable for irradiation at ORNL with internal volume $\sim 0.3 \mathrm{~mL}$. The containers were then held in an ultrasonicator bath without lids and sonicated until all the acetone was removed. Since neither $\mathrm{C}_{60}$ nor lutetium oxide are soluble in acetone, the sonication is another physical method of mixing. The $70 \%$ enriched ${ }^{176} \mathrm{Lu}$ samples were irradiated for only 2 minutes in PT-2, resulting in initial activities of $110 \mu \mathrm{Ci}$ each.

\subsection{Assessment of the Recoil Formation Yield of ${ }^{177}{ }^{2 u} @ C_{60}$}

At this point, the sample consisted of $\mathrm{C}_{60},{ }^{177} \mathrm{Lu} @ \mathrm{C}_{60}$, and $\mathrm{Lu}_{2} \mathrm{O}_{3}$ with a variety of $\mathrm{Lu}$ isotopes, including ${ }^{177} \mathrm{Lu}$. The next issue was to determine the portion of ${ }^{177} \mathrm{Lu}$ that was encapsulated in $\mathrm{C}_{60}$. Since both the 
${ }^{177} \mathrm{Lu} @ \mathrm{C}_{60}$ and the $\mathrm{Lu}_{2} \mathrm{O}_{3}$ are insoluble, we determined to first subject the entire sample to malonic esterification, as described in Section 3.2.1. As the esterification reaction renders the ${ }^{177} \mathrm{Lu} @ \mathrm{C}_{60}$ soluble, it provides a handle to separate the fullerenes from the oxide. Following the esterification reaction, the solids were removed, and the soluble portion was washed exhaustively with $0.12 \mathrm{M} \mathrm{HNO}_{3}$ containing $\sim 1 \times 10^{-6} \mathrm{M} \mathrm{Lu}^{+3}$ ions to remove any trace ${ }^{177} \mathrm{Lu}$ left in solution but outside of the fullerene. At this time, it was discovered that the yields were much less than reported by Braun and Rausch (1998), though there was unquestionably some ${ }^{177} \mathrm{Lu} @ \mathrm{C}_{60}$ implanted in the fullerene. Section 4.3.3 discusses why their data cannot possibly have been correct, and points to a group of relatively simple solutions that were partially explored, as reported in Section 4.3.4. We concluded that the recoil implantation method works well, but that the material to be irradiated must be prepared far more carefully than was reported by Braun and Rausch.

\subsubsection{Results of Malonic Ester Derivatization and Washing}

The results of exhaustive washing of the $2.0 \mathrm{mg}$ natural $\mathrm{Lu}_{2} \mathrm{O}_{3}-\mathrm{C}_{60}$ sample, derivatized to become an ester, are shown in Figure 19Figure . Clearly, the activity in the aqueous portion falls off rapidly, while the activity in the organic comes to an equilibrium after 6-7 washes. The activity in the fullerene portion levels off at about $3.0 \mathrm{nCi}$. Back-correcting this number to account for the decays occurring during time that sample was prepared shows that $6.0 \mathrm{nCi}$ were originally present in the fullerene. Since the sample originally contained $450 \mu \mathrm{Ci}$, however, the yield $\left({ }^{177} \mathrm{Lu}\right.$ basis $)$ is only $0.0013 \%$ ! This is far lower than we were anticipating, and severely lower than reported by Braun and Rausch in 1998.

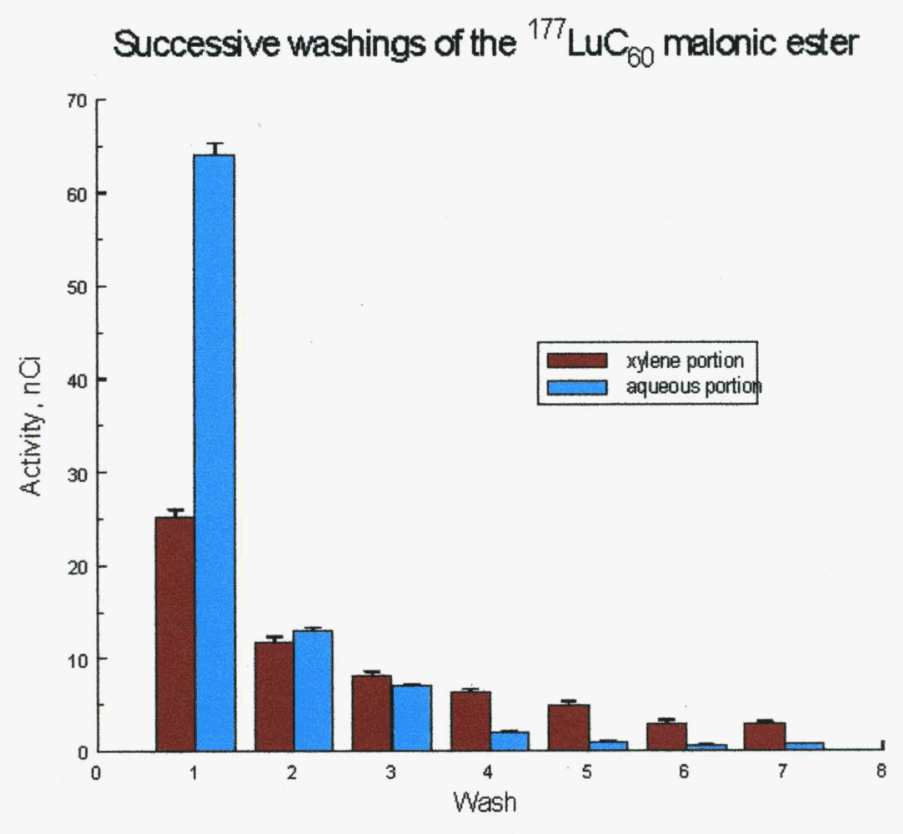

Figure 19: Washing the bucky ester with an aqueous Lu - nitric acid solution. Error bars are from the uncertainty in counting data. See text for details. 


\subsubsection{The Fundamental Problem with Recoil Formation of Radiofullerenes From Mixtures With Metal Oxides}

The explanation for the apparently low yield from the Lu oxide experiments lies in the lack of proximity between the fullerenes and most $\mathrm{Lu}$ atoms. We were trying to mix grains of $\mathrm{C}_{60}$ with grains of $\mathrm{Lu}_{2} \mathrm{O}_{3}$. A quick calculation demonstrates the problem.

Grains of metal oxides are typically between 1 and 10 microns in diameter. Since no special effort was made to make the $\mathrm{Lu}_{2} \mathrm{O}_{3}$ grains small, the assumption of a two micron grain is entirely reasonable. However, a heavy nuclide like Lu will only recoil a distance between 0.5 and $1 \mathrm{~nm}$ in a solid following a $(\mathrm{n}, \gamma)$ reaction (Lieser, 2001, for example). Again we will take the conservative case of the activated Lu nucleus recoiling $1 \mathrm{~nm}$. Therefore, it is only the atoms on the outside of the grain that have a chance of encountering a fullerene during recoil. Since the atoms inside of the grain are as likely to be activated as those on the outside, however, the portion of the activated nuclei that encounter a fullerene during recoil is only a small percentage of the nuclei that become activated.

Assuming spherical grains of $\mathrm{Lu}_{2} \mathrm{O}_{3}$ with diameter $2 \mu \mathrm{m}$, the portion of the $\mathrm{Lu}$ atoms in the outermost 1 $\mathrm{nm}$ shell can be calculated as the ratio of the volume of the outer $1 \mathrm{~nm}$ shell of the $2 \mu \mathrm{m}$ sphere to the volume of the $2 \mu \mathrm{m}$ sphere; i.e. $\left(1^{3}-.999^{3}\right) / 1^{3}=0.3 \%$. Thus, only $0.3 \%$ of the $\mathrm{Lu}$ atoms could recoil out of an average size oxide grain. However, grains tend to agglomerate, and physical mixing methods can not overcome this tendency. If we assume that $1 / 3^{\text {rd }}$ of the grain's surface interfaces with a fullerene, then only $0.1 \%$ of the Lu atoms even have a chance to encounter a fullerene during recoil.

If only one in a thousand ${ }^{177} \mathrm{Lu}$ atoms even had a chance to become trapped within a fullerene, then our yield calculated from the ratio of ${ }^{177} \mathrm{Lu}$ activity in the fullerene ester to the total ${ }^{177} \mathrm{Lu}$ activity $(0.0013 \%$, Section 3.3.3) in the sample does not appear so bad. We conclude that $1.3 \%$ of the activated Lu that could have encountered a fullerene became entrapped. Thus, if we could bring the $\mathrm{Lu}$ atoms and the fullerenes closer together, mixed on a molecular scale, then the yield could increase a thousand fold, into having a few ${ }^{177} \mathrm{Lu} @ \mathrm{C}_{60}$ per hundred ${ }^{177} \mathrm{Lu}$ atoms. Such a sample would generate a $10 \mathrm{mCi}$ dose while having only $1 \mathrm{Ci}$ total activity, and the other $990 \mathrm{mCi}$ is readily removed at the outset. Indeed, our result of $1-2 \%$ of the neutron-activated ${ }^{177} \mathrm{Lu}$ that had a chance to recoil into the fullerene forming an endohedral fullerene is very encouraging.

Though Braun and Rausch (1998) calculated 3\% or greater yields from the same experiment, with the same physical limitations, it is clearly impossible, and we can offer a possible explanation for their error. Following irradiation, they extract the fullerene sample with aniline. The primary amine functionality on aniline readily forms charge-transfer complexes with a wide variety of electron-poor materials, including empty fullerenes, metallofullerenes, and possibly hot lanthanide ions that lost electrons as the gamma ray was emitted. Common chelators for radionuclides (EDTA, DTPA, DOTA, etc.) are after all just amines separated by ethyl groups. Thus they observe a lot of activity in the aniline, even though most of the activity is not in a fullerene, but "chelated" by the aniline amine. They then extract the sample with acid, but there is no cold Lu in the acid. Without cold lanthanides in the wash, there is no exchange between hot and cold lanthanides, and the aniline retains its hold on the hot lanthanide. It is therefore not surprising that they found no hot lanthanide in the acid part of the wash. Whether this is the reason or not, as they may have made other errors impossible to discern without directly observing their experiments, their claim of $3 \%$ yield from mechanical mixing of lanthanide oxides and $\mathrm{C}_{60}$ turns out to be absolutely impossible. Fortunately, there are ways to fix this fundamental problem, specifically by avoiding oxides and by mixing by chemical instead of physical means. 


\subsubsection{Avoiding the Fundamental Problem: Preparation and Activation of Non-oxide $\mathbf{L u}-\mathrm{C}_{60}$ Mixtures}

We first tried to improve the mixing by co-precipitating $\mathrm{C}_{60}$ and $\mathrm{Lu}\left(1,2\right.$-pentanedionate) ${ }_{3}$ [also known as $\mathrm{Lu}(\mathrm{acac})_{3}$ ] from various solvents. Both are soluble in xylene, and a mixture of $2 \mathrm{mg}$ each acac and $\mathrm{C}_{60}$ was stirred into $4 \mathrm{~mL}$ xylene. The xylene was then removed under flowing gas down to the last $0.5 \mathrm{~mL}$, which was transferred into a polyethylene activation container. The final xylene was removed with flowing gas, but the result was unsatisfying: the upper wall of the container was coated with a black substance, presumably the $\mathrm{C}_{60}$, while lower part of the container wall was coated with a light brown material, presumably the acac.

Though the solubility differences in xylene were too large for a co-precipitation method to work, perhaps a different soluble would be more suitable. Two $\mathrm{mg}$ of both $\mathrm{C}_{60}$ and the acac were then dissolved in dimethylformamide (DMF) and stirred. The DMF was then removed under vacuum, although it is very difficult to completely remove the high-boiling DMF. The flask was scraped, and the recovered sludge was placed in a sample container. The sludge was activated in PT-2 for $20 \mathrm{~min}$, and left to cool for a day.

The conversion to ester was the same as described for the oxides, and three washes were performed. Figure 20 shows the activity after various steps of the sample work-up procedure. This sample is remarkable versus the oxides because $66 \%$ of the activity in the flask prior to ester derivatization was initially soluble in toluene. For the oxides, $0.1 \%$ or less remained in solution following centrifugation. A set of three washings removed an additional $50 \%$ of the activity in the flask prior to derivatization, with the final wash not being particularly productive.

Of course, we expect $\sim 99 \%$ of the sample activity to be outside of the fullerene under the best possible mixing conditions. Of the $34.6 \mu \mathrm{Ci}$ transferred form the container into the flask, only $\sim 0.35 \mu \mathrm{Ci}$ at most would be expected in the fullerene. Yet after washing, $5.82 \mu \mathrm{Ci}$ remains in solution. Either the acac ligands are able to hold onto the $\mathrm{Lu}$ atoms following recoil, or the remaining DMF forms charge transfer complexes with the free $\mathrm{Lu}$ ions. If the latter case was true, and radioactive lanthanides cannot be washed out of a charge-transfer complex with a secondary amine (DMF), then Braun and Rausch had no hope of washing radioactive lanthanides from charge

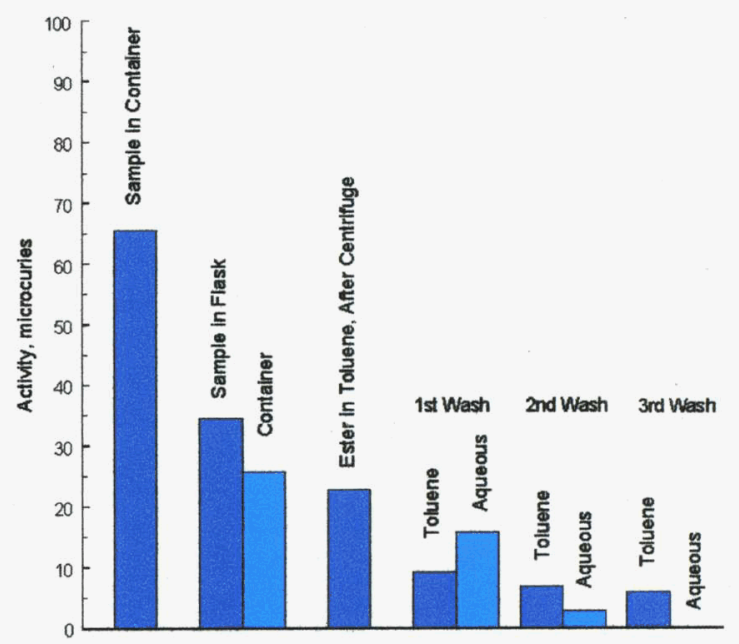

Figure 20: Chart of the activity during the work-up of the $\mathrm{Lu}(\mathrm{acac})-\mathrm{C}_{60}$-DMF sample. The sum of the toluene and aqueous activities following a wash is $85-109 \%$ of the toluene solution prior to that wash. transfer complexes with a primary amine (aniline). It is difficult to assess the recoil implantation efficiency from this technique, without knowing the chemical state of so much of the ${ }^{177} \mathrm{Lu}$. HPLC analysis of this sample, described in Section 3.4, would reveal that $0.11 \%$ of the injected portion would elute from the column with the fullerenes. Though some of the radiofullerene ester would undoubtedly be lost to active sites on the column, using the $0.11 \%$ As a lower bound for the portion of the $5.82 \mu \mathrm{Ci}$ that is contained in the fullerene gives an overall yield of $(.0011 * 5.82 / 34.6)=0.018 \%$, a tenfold improvement over the oxides.

Though clearly on the right track, the DMF method could still be less than optimum for several reasons. Even though the sludge appears uniform, microcrystals of $\mathrm{Lu}(\mathrm{acac})$ and $\mathrm{C}_{60}$ could still form during evaporation, similar to the experience with xylene. It is also possible that the DMF forms charge-transfer 
complexes with $\mathrm{C}_{60}$ and/or Lu@ $\mathrm{C}_{60}$. Though these ought to be broken by the action of the hydride on the $\mathrm{C}_{60}$, it has never been studied. Significantly reducing the esterification reaction yield would appear as a reduction in recoil implantation yield in our calculation, since we are calibrating off of the portion that eluted through the HPLC as an ester. We have by no means exhausted the list of solvents potentially suitable for co-precipitation mixing of $\mathrm{C}_{60}$ and $\mathrm{Lu}(\mathrm{acac})$, and the encouraging result versus the oxide suggests that further work is warranted.

It is possible that the optimum solution was to mix the fullerene with the lutetium at and atomic level, i.e. prepare a $\mathrm{Lu}_{\mathrm{x}} \mathrm{C}_{60}$ salt. Fullerene salts of certain lanthanides are known, specifically those with $\mathrm{Sm}$ (Chen and Roth, 1995), Eu (Ksari-Habiles et al., 1997), and Yb (Ozdas et al., 1995). These compounds are either superconductors $(\mathrm{Sm}, \mathrm{Yb})$ or ferromagnetic (Eu). Given their interesting solid state properties, it is, at first glance, surprising that no other lanthanide salts have been reported. The reason is that, amongst lanthanides, $\mathrm{Sm}, \mathrm{Eu}$, and $\mathrm{Yb}$ have by far the greatest vapor pressure (Figure 21Figure ) and therefore most prone to undergo solid state reactions. These salts were prepared by simple heating with $\mathrm{C}_{60}$ at ca. $600 \mathrm{C}$, with reaction times varying between hours and weeks. All authors report a narrow temperature range through the reaction occurs without decomposing the $\mathrm{C}_{60}$. Sadly, $\mathrm{Lu}$ has a rather low vapor pressure, and the $\mathrm{C}_{60}$ would decompose at any temperature when the reaction proceeds at a measurable rate.

Other fulleride salts have been prepared with Group I and II metals, since they also have high vapor pressure (relative to other metals). These have also found to be superconducting, sparking a flurry in interest in their preparation. Electrochemical methods are prone to contamination by counter ions, which usually contain halides, which are forbidden from the HFIR's pneumatic tubes. However, Chen et al. (1997) offer an interesting preparation of $\mathrm{Rb}_{3} \mathrm{C}_{60}$ at room temperature. The authors mixed $\mathrm{C}_{60}$ powder and $\mathrm{Rb}$ powder in a

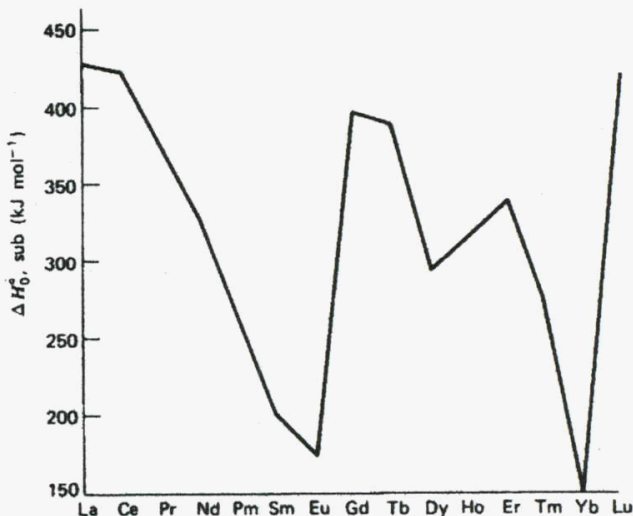

Figure 21: Enthlapies of Sublimation for the Lanthanides (Cotton and Wilkinson, 1988). flask with propylamine as the sole liquid. The propylamine readily forms charge transfer complexes with the $\mathrm{C}_{60}$, and a tell-tale green solution. This complex is subject to reduction by the $\mathrm{Rb}$, forming an $\mathrm{Rb}-\mathrm{C}_{60}$ salts which precipitates. The amine is then removed under vacuum.

Although $\mathrm{Lu}$ is $\sim 0.6 \mathrm{~V}$ less powerful a reducing agent than $\mathrm{Rb}$, we thought to try this reaction. In the dry box, $36 \mathrm{mg} \mathrm{C} 60$ and $8 \mathrm{mg} \mathrm{Lu}$ ( $40 \mathrm{mesh}$ ) were added to a 3-neck $50 \mathrm{~mL}$ flask. The flask was removed to a Schlenk line, to which $25 \mathrm{~mL}$ of degassed propylamine was added, and the reaction stirred vigorously. We also thought to try a tertiary amine, which might be easier to remove. To that end, $\mathrm{C}_{60}$ and Lu were added to DBU in the dry box. Both reactions took on a green color within hours. After several days, metallic Lu could still be seen at the bottom of both flasks. However, the propylamine reaction had lost its green color, becoming brownish-red, as predicted by Chen et al. The DBU reaction remained green, and was not pursued further.

The propylamine was removed under vacuum, and the recovered solids were split in two to create two samples. They were both irradiated for $20 \mathrm{~min}$ in PT-2. After several days' worth of cooling off, their gamma ray spectrum was similarly clean as that in Figure 22Figure. Addition of the THF/NaH slurrly, however, resulted in a green solution, some of which was set aside. The THF of the set aside portion was removed, and xylene was added, creating a brown solution reminiscent of the ester. Washing of the THF portion caused a precipitate, while washing the xylene was rather slow, but ultimately resulted in complete loss of activity from the organic. In summary, the presence of leftover propylamine, with 
unclear charge states on the fullerenes, caused the esterification reaction to veer off, at least partially, into unknown products.

Probably the optimum method to prepare ${ }^{177} \mathrm{Lu} @ \mathrm{C}_{60}$ is to mix isotopically enriched ${ }^{176} \mathrm{Yb}$ with $\mathrm{C}_{60}$, heat to $600 \mathrm{C}$ to form the $\mathrm{Yb}_{\sim 3} \mathrm{C}_{60}$ salt (after Ozdas et al., 1995), and activate that salt. The reaction ${ }^{176} \mathrm{Yb}(\mathrm{n}, \gamma){ }^{177} \mathrm{Yb}(\beta, \gamma){ }^{177} \mathrm{Lu}$ is in fact the common process to make ${ }^{177} \mathrm{Lu} .{ }^{177} \mathrm{Yb}$ has a half-life of only $1.9 \mathrm{~h}$, so high ${ }^{177} \mathrm{Lu}$ radionuclidic purity is readily obtained. The issue however, is that the neutron cross section of ${ }^{176} \mathrm{Yb}$ is only $2.4 \mathrm{~b}$, requiring a fairly long irradiation time (or higher neutron flux) to acquire much activity.

To summarize this section, radionuclides can be implanted in $\mathrm{C}_{60}$ by recoil following neutron activation and derivatized via multiple malonic ester addition reactions to become soluble in a wide variety of organic solvents. However, the implantation occurred at rather low yields, a result due entirely to the preparation of the cold material. Unless the cold atoms to be activated are within a few nanometers of a fullerene cage, there is essentially no chance for them to be encapsulated via recoil. Physical mixing of oxides and fullerenes, despite a report in the literature of high yields, allows only extremely poor encapsulation efficiencies $(\sim 0.001 \%)$. Fortunately, the fundamental mixing problem is solvable, and probably solvable by a variety of methods, including co-precipitation of $\mathrm{C}_{60}$ and organometallics, formation of metal- $\mathrm{C}_{60}$ salts, and co-deposition of $\mathrm{C}_{60}$ and organometallic vapors on a substrate under vacuum. We have already observed a 10 -fold increase in encapsulation efficiency by the most rudimentary of these techniques (co-precipitation), and believe that an efficiency of $>1 \%$ would be rapidly attainable with continued effort.

\subsection{HPLC Separation of ${ }^{177} \mathrm{Lu} @ \mathrm{C}_{60}$ Bingel Ester from Cold $\mathrm{C}_{60}$ Bingel Esters}

Although the malonic ester derivative of the ${ }^{177} \mathrm{Lu} @ \mathrm{C}_{60}$ is now in hand, it is but a tiny portion of all the fullerene esters in solution. It must be purified before it is suitable for conjugation to an antibody (Ab). Unlabeled $\mathrm{mAbs}$ or $\mathrm{mAbs}$ that are chelated with non-radioactive atoms are essentially "shooting blanks". The final radiofullerene complex (before conjugation to the $\mathrm{mAb}$ ) should contain at least $10 \%$ radioactive atoms to be competitive with current radiotherapy techniques. However, this is only a guide; lower specific activity may be satisfactory depending on the specific therapy and advantage gained by using the fullerene. Therefore, we needed to demonstrate that the radiofullerenes could be separated from the cold fraction.

We had originally considered exhaustive washing of the activated fullerene material prior to derivatization. Since $\mathrm{Ln} @ \mathrm{C}_{60}(\mathrm{Ln}=$ lanthanide $)$ is insoluble in aromatic solvents such as xylene, while empty $\mathrm{C}_{60}$ is soluble, there appears to be the basis for a separation. Additional thought, however, suggests that the tiny percentage of $\mathrm{Ln} @ \mathrm{C}_{60}$ would more than likely be merely carried along in the solvent, perhaps in fullerene micelles. There are no regions of $\mathrm{Ln} @ \mathrm{C}_{60}$; no nanocrystals from which the surrounding material can be washed away. The $\mathrm{Ln} @ \mathrm{C}_{60}$ is intermolecularly mixed with the empty $\mathrm{C}_{60}$. The odds are strongly against the solvent selectively removing all fullerenes round the endohedral one, while letting the endohedral fullerene settle to the bottom of the flask.

Fortunately, the chemical differences between empty and lanthanide-filled fullerenes manifest themselves in the number of malonic ester groups that add to the exterior of fullerene cage. In the reaction conditions employed here, empty fullerenes add a mixture of 4,5 , or 6 malonic ester groups. It is known that adding a lanthanide atom to $\mathrm{C}_{60}$ increases the number of malonic ester ligands to 10 when subjected to the same conditions (Bolskar et al., 2003). The varying number of malonic esters on the fullerene shell provides a means to chromatographically separate the endohedral fullerene esters from the empty fullerene esters. 
Our approach to this problem was to search for a chromatographic method suitable for the separation of the 4,5 , and 6 esters, reasoning that such a method would also be sufficiently powerful to separate the 10 ester endohedral fullerene from the others. We initially predicted that a mobile phase of methanol with a little xylene in it over a reverse phase $(\mathrm{C} 18)$ column would provide the necessary separation. While it provided separation into two distinct peaks, which were collected as fractions, the TOF-MS analysis of the fractions revealed that the esters had each lost 155 mass units. TOF-MS analysis of the ester dissolved in methanol (it is very slightly soluble) and filtered did not display such action, and neither the rationale for nor the identity of the fragment have been determined. In any case, a different method would be required.

While using hexane to cut the toluene did not slow the esters from rushing straight over the $\mathrm{C} 18$ column, a one-to-one mixture provided both retention and separation on our semi-prep Buckyclutcher column. Moreover, TOF-MS of the collected fractions was able to identify each of the three peaks as corresponding to one of the esters. Their elution order was 4, 5, and then 6 . Using the photodiode array detector (PDA) at ORNL, the uv-vis spectrum of each of the esters was observed as it eluted. The spectra, shown in Figure 22Figure, have distinctive features that allow us to identify them without further TOF-MS analysis. That the features exist perhaps suggest that, as the $\mathrm{C}_{60}$ becomes crowded, not so many isomers of a particular composition are possible.
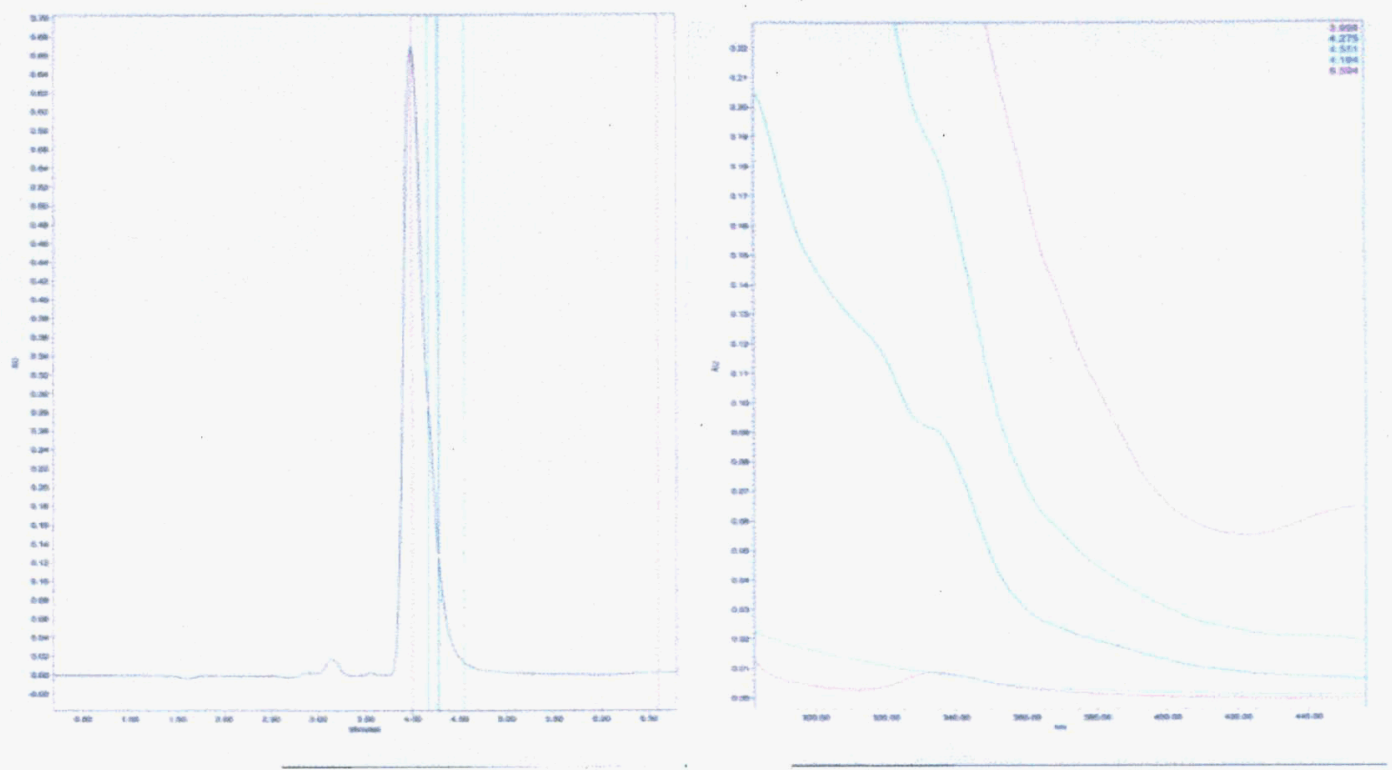

Figure 22: Left: Absorbance at $310 \mathrm{~nm}$ of a mixture of empty $\mathrm{C}_{60}$ with 4, 5, and 6 malonic esters in toluene chromatographed on a semi-prep BuckyPrep column with $3 \mathrm{~mL} / \mathrm{min}$ each toluene and hexane. Right: UV - vis absorbance at times corresponding to the vertical lines of the same color in the chromatogram, i.e. the double-humped dark blue spectrum elutes after the single-humped light blue, which is after the red with the absorbance in the visible. The two spectra with little absorbance are the last two vertical lines in the chromatogram. 
Predicting that the 10-ester $\mathrm{Lu} @ \mathrm{C}_{60}$ would elute far later than the empty fullerenes with 4,5 , or 6 esters, we injected the mixture of hot and cold malonic ester derivatives using a flow of $3 \mathrm{~mL} / \mathrm{min}$ each toluene and hexane over the BuckyPrep column. We collected fractions every 30 seconds starting from injection, and counted the fractions individually on the Wallac Wizard.

The results of the toluene/hexane hot ester separation are given in Figure 23. In an effort to increase the counts, we saturated the detector in the uv with cold ester, making it appear that we failed to achieve separation between the 4,5 , and 6 esters. Nevertheless, spectra taken at various times within the large unresolved peak in Figure 23 show similar uv-vis as those in Figure 22Figure. The plot of activity in each fraction, however, is hard to completely understand. The little bit of activity eluting prior to 2 minutes is indicative of a small amount of the ${ }^{177} \mathrm{Lu} @ \mathrm{C}_{60}$ rushing through with the pure toluene it was injected in, the so-called "injection peak." This is to be expected. The large amount of activity eluting between 2.5 and $3.5 \mathrm{~min}$ is very difficult to understand in the context of the empty fullerene ester HPLC work, and the cold metallofullerene ester TOF-MS work. That some ${ }^{177} \mathrm{Lu} @ \mathrm{C}_{60}$ ester would elute with the empty fullerene esters between 4 and $5.5 \mathrm{~min}$ is perhaps also reasonable if the empty fullerene esters solvate, to some extent, the hot fullerene ester. Lastly, some activity seems to dribble off the column from six minutes on. Satisfyingly, over $40 \%$ of the injected activity eluted. We were concerned that active sites within the column would cause the tiny amount of hot fullerene ester to retained on the column, since its different chemistry precludes the empty fullerenes from acting as true carriers. So it was very encouraging that a large portion of the ${ }^{177} \mathrm{Lu}$ was briefly retained on the column, and then eluted, more or less like the fullerene ester in which we expect it to be irreversibly encapsulated. Arguments that the ${ }^{177} \mathrm{Lu}$ is not really in the fullerene (if any could be left after the washing exercise) are dispelled by this result. However, it also appeared that a different chromatographic method would be required to effectively separate and collect the ${ }^{177} \mathrm{Lu} @ \mathrm{C}_{60}$.

Since the BuckyPrep stationary phase has excellent selectivity for the fullerene, we reasoned that the mobile phase should be directed towards the malonic ethyl esters. Thus, we selected ethylacetate for the mobile phase. An injection of cold esters into pure ethyl acetate produced a trio of partially resolved peaks, as well as a leading peak that absorbs moderately in the uv, and in invisible in the visible spectral region. The chromatogram and the uv-vis of the major peaks in the chromatogram are shown in Figure 24. Interestingly, in comparison to the spectra obtained with toluene/hexane (Figure 22), the elution order of the esters is reversed. With ethylacetate as the mobile phase, fullerenes with six malonic esters, as identified by uv-vis and TOF-MS, elute first. The fullerenes with five malonic esters are still in the middle, and those with 4 elute last. Thus, in ethylacetate, we expect the Lu metallofullerenes to be first off of the column.

The merits of the ethylacetate-BuckyPrep chromatographic method were initially assessed with the ester formed from neutron activation of $\mathrm{Lu}(\mathrm{acac}) / \mathrm{C}_{60}$ mixtures in DMF (Section 4.3.3). As can be seen in Figure 25 , the activity eluted slightly before the empty fullerene peaks, exactly as expected. Obviously, some tailing under or co-solvation with the empty fullerenes occurs, but further development of the this method can be expected to solve these problems. This result was later repeated with ${ }^{177} \mathrm{Lu} @ \mathrm{C}_{60}$ esters. 

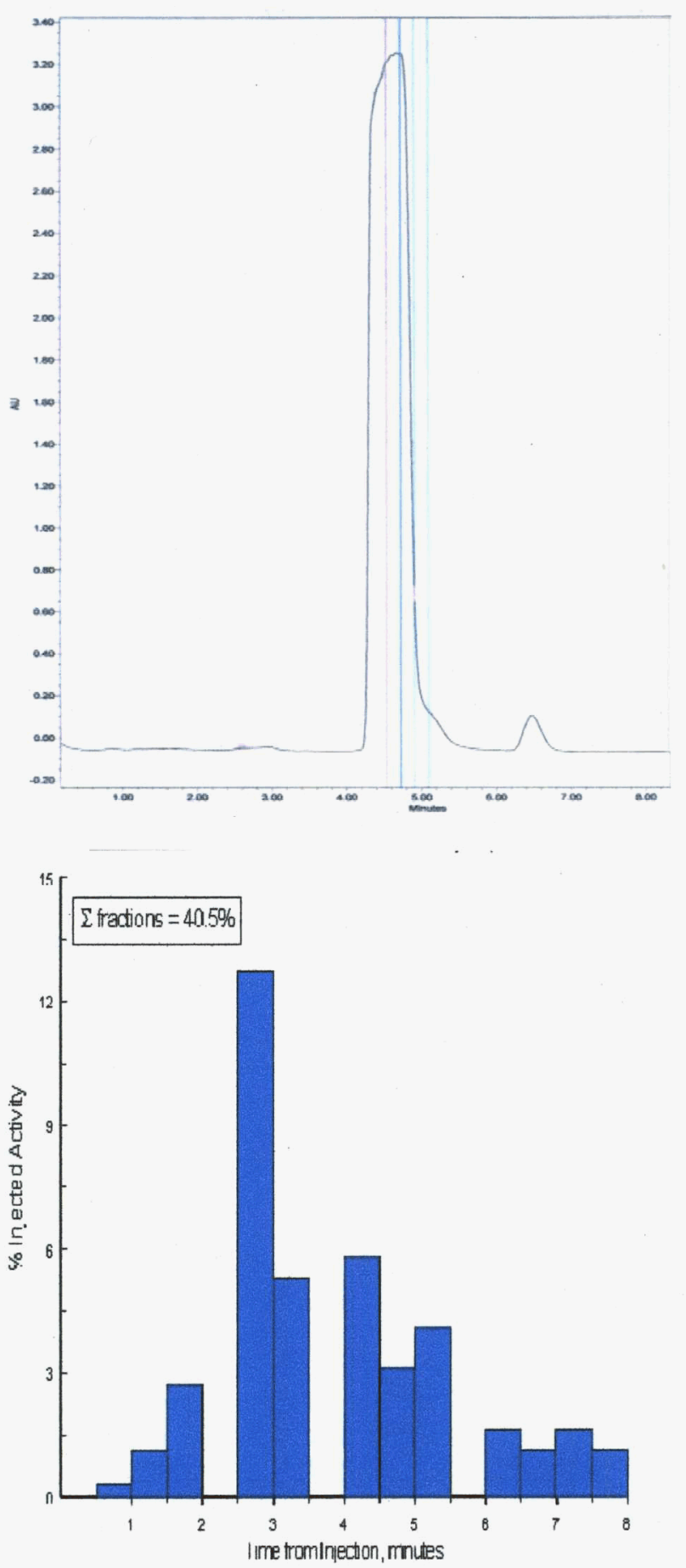

Figure 23: Chromatogram of ${ }^{176} \mathrm{Lu}$ oxide $+\mathrm{C}_{60}$ mixture, derivatized by the Bingel procedure, loaded onto the BuckyPrep column, and eluted with $3 \mathrm{ml} / \mathrm{min}$ toluene $+3 \mathrm{ml} / \mathrm{min}$ hexane. Top: uv absorbance @ $310 \mathrm{~nm}$. Bottom: ${ }^{177} \mathrm{Lu}$ activity in fractions collected every 30 seconds from injection. 

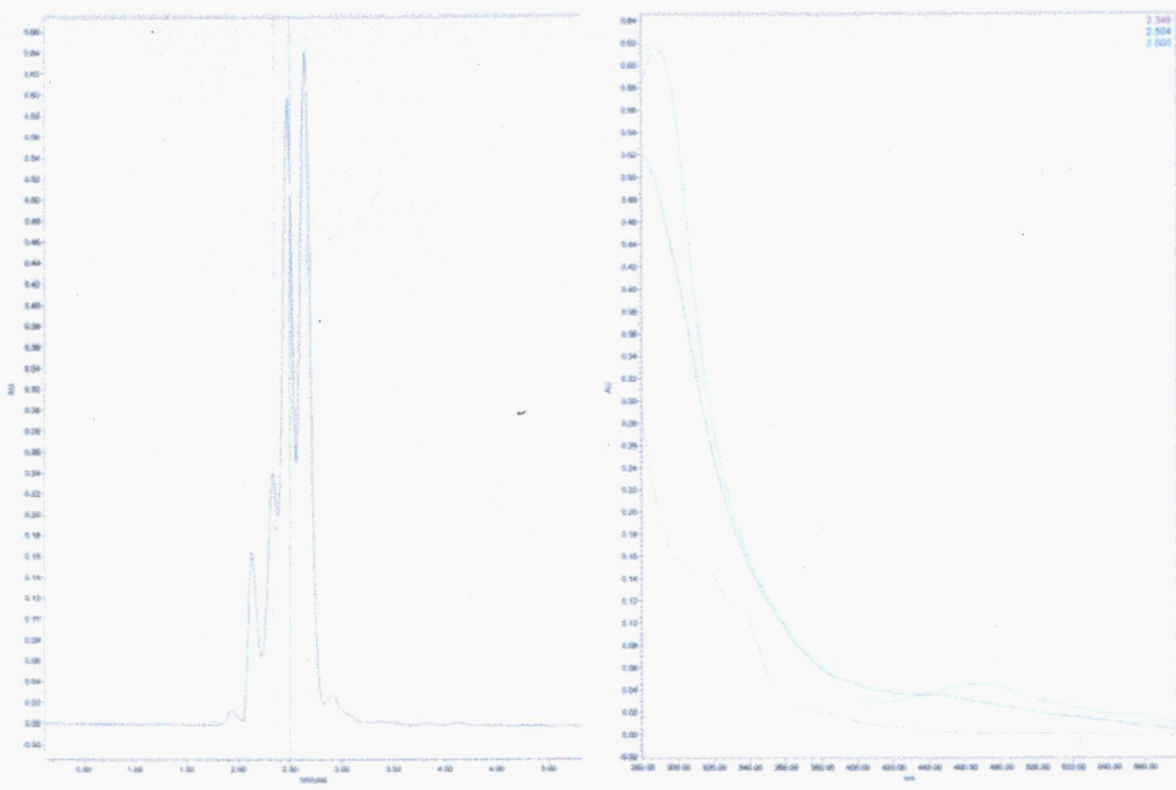

Figure 24: Left: Absorbance at $310 \mathrm{~nm}$ of a mixture of empty $\mathrm{C}_{60}$ with 4, 5, and 6 malonic esters in toluene chromatographed on a semi-prep BuckyPrep column with $6 \mathrm{~mL} / \mathrm{min}$ ethylacetate. Right: UV vis absorbance at times corresponding to the vertical lines of the same color in the chromatogram, i.e. the double-humped red spectrum elutes before the weak single-humped dark blue, which is before the light blue with the absorbance in the visible $(470 \mathrm{~nm})$. 


\subsection{Summary of ${ }^{177} \mathbf{L u} @ \mathrm{C}_{60}$ Studies}

The primary achievement of this part of the project was that incontrovertible evidence for the production of ${ }^{177} \mathrm{Lu} @ \mathrm{C}_{60}$ via recoil implantation following neutron activation was obtained. We also established a rapid protocol for the chemical derivatization of the hot endohedral fullerene, leading to a robust, nonaggregating water-soluble radiofullerene derivative. Furthermore, we developed a chromatographic technique suitable for its fast, convenient purification from cold, derivatized fullerenes. The chromatography will allow for arbitrarily high specific activity of ${ }^{177} \mathrm{Lu}$-containing fullerenes to be obtained, limited only by the time between activation and separation. Although the (neutron capture) recoil implantation yield was not as high as originally expected, the reason for the discrepancy is understood, and solutions have been identified. In summary, we have demonstrated the promise of the recoil implantation technique for producing radiofullerenes suitable for pharmaceutical development, met only readily surmountable hurdles, and remain enthusiastic about the continued development of the technique.

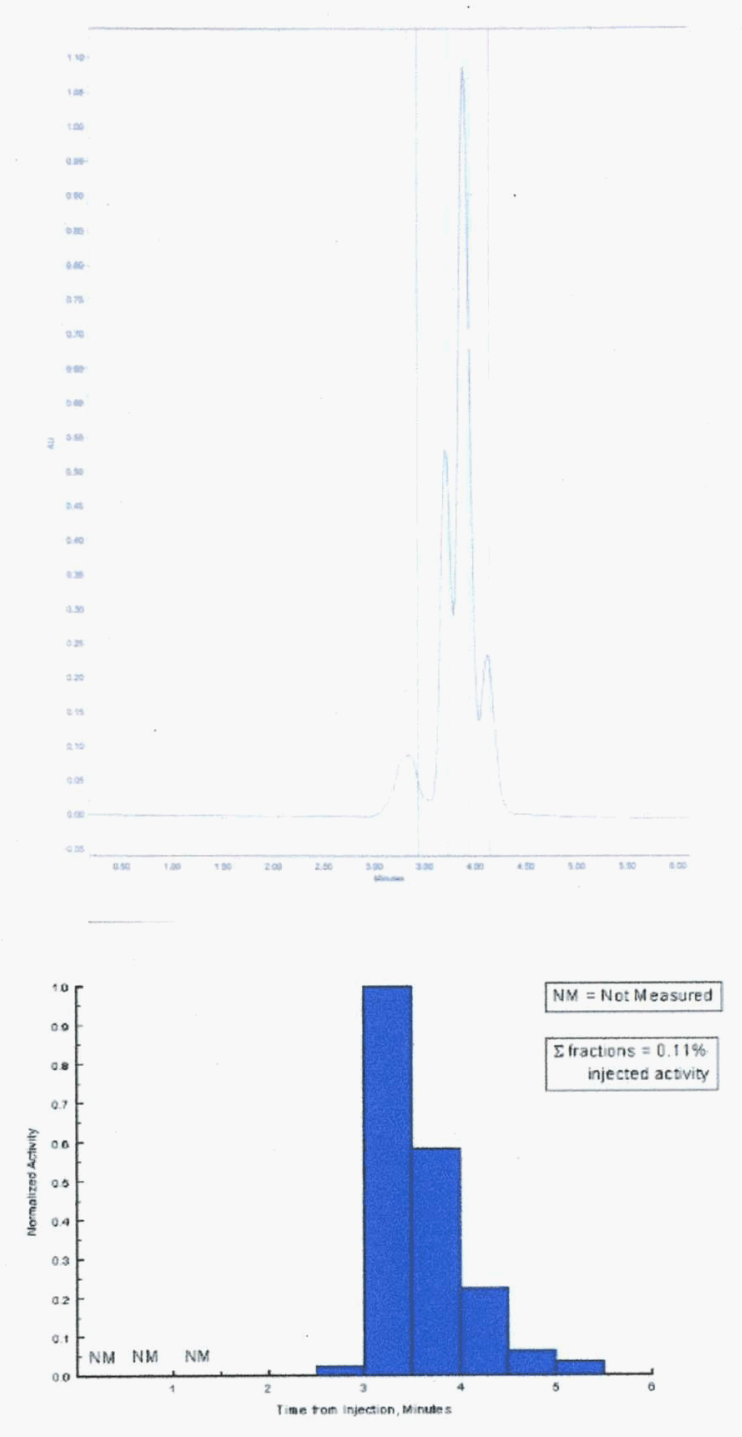

Figure 25: Chromatogram of $\mathrm{Lu}(\mathrm{acac})-\mathrm{C}_{60}$ mixture, coprecipitated from DMF, activated, derivatized by the Bingel procedure, loaded onto the BuckyPrep column, and eluted with $4 \mathrm{~mL} / \mathrm{min}$ ethyl acetate $+2 \mathrm{~mL} / \mathrm{min}$ hexane. Top: uv absorbance @ 310nm. Bottom: ${ }^{177} \mathrm{Lu}$ activity in fractions collected every 30 seconds from injection. 


\section{References}

Adams, G.P., et al. (2000). "Delivery of the $\alpha$-emitting Radioisotope Bismuth-213 to Solid Tumors via Single-Chain Fv and Diabody Molecules," Nuc. Med. Biol. 27, 339-346.

Alvarez, R.D., E. E. Partridge, M. B. Khazaeli, G. Plott, M. Austin, L. Kilgore, C. D. Russell, W. E. Grizzle, J. Schom, A. F. LoBuglio and R. F. Meredith, (1997). "Intraperitoneal Radioimmunotherapy of Ovarian Cancer with 177Lu-CC49: A Phase I/II Study," Gynecologic Oncology, 65, 94-101.

Akiyama, K. et al. (2001). "Isolation and Characterization of Light actinide Metallofullerenes," J. Am. Chem. Soc. 123, 181-2.

Bolskar, R.D. et al., (2003). "First Soluble M@C 60 Derivatives Provide Enhanced Access to Metallofullerenes and Permit in Vivo Evaluation of $\mathrm{Gd} @ \mathrm{C}_{60}\left[\mathrm{C}(\mathrm{COOH})_{2}\right]_{10}$ as a MRI Contrast Agent," JACS 125, 5471-8.

Borchardt, P.E., R.R. Yuan, M. Miederer, M.R. McDevitt, and D.A. Scheinberg (2003). "Targeted Actinium-225 in Vivo Generators for Therapy of Ovarian Cancer," Cancer Res. 63, 5084.

Braun, T. and H. Rausch, (1995). "Endohedral Incorporation of Argon Atoms Into C 60 by Neutron Irradiation," Chem. Phys. Lett. 237, 443-7.

Braun, T. and H. Rausch, (1998). "Radioactive endohedral metallofullerene formed by prompt gammagenerated nuclear recoil implosion", Chem. Phys. Lett. 288, 179.

Cagle, W. D., S. J. Kennel, S. Mirzadeh, J. M. Alford, and L. J. Wilson, (1999). "In vivo Studies of Fullerene-based Materials Using Endohedral Metallofullerene Radiotracers," Proc. Natl. Acad. Sci. USA 96, 5182.

Cagle, D. W., T. P. Thrash, J. M. Alford, L. P. F. Chibante, G. J. Ehrhardt and L. J. Wilson, (1996). "Synthesis, Characterization, and Neutron Activation of Holmium Fullerenes", J. Am. Chem. Soc. $118,8043$.

Chai, Y., T. Guo, C. Jin, R. E. Haufler, L. P. F. Chibante, J. Fure, L. Wang, J. M. Alford, and R. E. Smalley (1991). "Fullerenes with Metals Inside," J. Phys. Chem. 95, 7564.

Chen, X. et al., (1997). "Preparation of $\mathrm{Rb}_{3} \mathrm{C}_{60}$ Utilizing Room-Temperature Solubiliaztion in Alkylamines," Chem. Mater. 9, 3049-3051.

Chen, X.H. and G. Roth (1995). "Superconductivity at 8K in Samarium-Doped C60," Phys. Rev. B 52, 15534-6.

Chong, H-S. , et al. (2006). "In vitro and in vivo Evaluation of Novel Ligands for Radioimmunotherapy," Nuc. Med. Biol. 33, 459-467.

Chappell, L.L. et al., (2000). "Synthesis, Chracterization, and Evaluation of a Novel Bifunctional Chelating Agent for the Lead Isotopes ${ }^{203} \mathrm{~Pb}$ and ${ }^{212} \mathrm{~Pb}$," Nuc. Med. Biol. 27, 93.

Cotton, F.A. and G. Wilkinson (1988). Advanced Inorganic Chemistry, $5^{\text {th }}$ ed., Wiley Interscience, 962.

Couturier, O. et al. (2005). "Cancer Radioimmunotherapy with Alpha-Emitting Nuclides," Eur. J. Nuc. Med. Mol. Imag. 32, 601-614.

Davis, I.A., K.A. Glowienka, R.A. Boll. K.A. Deal. M.W. Brechbiel, M.Stabin, P.N. Bochsler, S. Mirzadeh, and S.J. Kennel, (1999). "Comparison of ${ }^{225}$ Actinium Chelates: Tissue Distribution and Radiotoxicity”, Nuc. Med \& Biol. 26, 581-589.

Deal, K.A., I.A. Davis, S. Mirzadeh, S.J. Kennel, and M.W. Brechbiel, (1999). "Improved In Vivo Stability of Actinium-225 Macrocyclic Complexes", J. Med. Chem. 42, 2988-2992.

Diener, M. D. and J. M. Alford, (1998). "Isolation and Properties of Small-Bandgap Fullerenes," Nature $393,668$.

Diener, M.D., C.A. Smith, and D.K. Veirs (1997). “Anaerobic Preparation and Solvent Free Separation of Uranium Endohedral Metallofullerenes,” Chem. Mater. 9, 1773.

Dugan, L.L., E. G. Lovett, K. L Quick, J. Lotharius, T.T. Lin, and K.L. O'malley (2001). "Fullerenebased antioxidants and neurodegenerative disorders", Parkinsonism and Related Disorders 7, 243. 
Dugan, L. L., J. K. Gabrielsen, S. P. Yu, T.-S. Lin, and D. W. Choi, (1996). "Buckminsterfullerenol Free Radical Scavengers Reduce Excitotoxic and Apoptotic Death of Cultured Cortical Neurons," Neurobiology of Disease 3, 129-135.

Dugan, L. L., D. M. Turetsky, C. Du, D. Lobner, M. Wheeler, C. R. Almli, C. K.-F. Shen, T.-Y. Luh, D. W. Choi and T.-S. Lin, (1997). "Carboxyfullerenes as Neuroprotective Agents," Proc. Natl. Acad. Sci. 94, 9434-9439.

Gadd, G. E., P. J. Evans, D. J. Hurwood, P. L. Morgan, S. Moricca, N. Webb, J. Holmes, G. McOrist, R. Wall, M. Blackford, D. Cassidy, M. Elcombe, J. T. Noorman, P. Johnson and P. Prasad, (1997). "Endohedral Fullerene Formation Through Prompt Gamma Recoil," Chem. Phys. Lett. 270, 108-114.

Gadd, G. E., P. Schmidt, C. Bowles, G. McOrist, P. J. Evans, J. Wood, L. Smith, A. Dixon and J. Easey, (1998). "Evidence for Rare Gas Endohedral Fullerene Formation from $\square$ Recoil from HPLC Studies," J. Am. Chem. Soc. 120, 10322-10325.

Gromov, A., D. Ostrovskii, A. Lassesson, M. Jonsson, and E.E.B. Campbell (2003). "Fourier Transform Infrared and Raman Spectroscopic Study of Chromatographically Isolated Li@ $\mathrm{C}_{60}$ and $\mathrm{Li}_{2} @ \mathrm{C}_{60}, " J$. Phys. Chem. B 107, 11290.

Guo, T., M.D. Diener, Y. Chai, J.M. Alford, R.E. Haufler, S.M. McClure, T. Ohno, J.H. Weaver, G.E. Scuseria, and R.E. Smalley (1992). "Uranium Stabilization of $\mathrm{C}_{28}$ : A Tetravalent Fullerene," Science 257, 1661.

Hassfjell, S. and M.W. Brechbiel (2001). "The Development of the $\alpha$ Particle Emitting Radionuclides ${ }^{212} \mathrm{Bi}$ and ${ }^{213} \mathrm{Bi}$, and the Their Deay Chain Related Radionuclides, for Therapeutic Applications," Chem. Rev. 101, 2019.

Heath, J. R., S. C. O'Brien, Q. Zhang, L. Liu, R. F. Curl, H. W. Kroto, F. K. Tittel, and R. E. Smalley, (1985). "Lanthanum Complexes of Spheroidal Carbon Shells," J. Am. Chem. Soc. 107, 7779-7780.

Hirsch, A., (1994). The Chemistry of the Fullerenes, Georg Thieme Verlag Publ., New York.

Hirsch, A. (1993). Angew. Chem., Int. Ed. Engl. 32, 1138-1141.

Horak E. et al (1997). "Radioimmunotherapy Targeting of HER2/neu Oncoprotein on Ovarian Tumor Using Lead-212-DOTA-AE1," J. Nuc. Med. 38, 1944.

Huang, H. and S. Yang (2000). "Preparation and Characterization of the Endohedral Metallofullerene Lu@C C, "J. Phys. Chem. Solids 61, 1105-1110.

Illidge, T. M., and S. Brock, (2000). "Radioimmunotherapy of Cancer: Using Monoclonal Antibodies to Target Radiotherapy," Current Pharmaceutical Design 6, 1399-1418.

Jaggi, J.S. et al. (2005). "Efforts to Control the Errant Products of a Targeted In Vivo Generator," Cancer Res. 65, 4888-4895.

Jensen, A. W., S. R. Wilson, and D. I. Schuster, (1996). "Biological Applications of Fullerenes: A Review," Bioorganic Chemistry 4 767-779.

de Jong, M. et al. (2001). " [ ${ }^{177}$ Lu-DOTA ${ }^{0}$, TYR $\left.^{3}\right]$ Octreotate for Somatostatin Recpetor-Targeted Radionuclide Therapy," Int. J. Cancer 92, 628-633.

Jurcic, J.G. et al. (2002). "Targeted $\alpha$ Particle Immunotherapy for Myeloid Leukemia," Blood 100, 1233.

Kennel, S.J., L.L. Chapell, K. Dadachova, M.W. Brechbiel, T.K. Lankford, I.A. Davis, M. Stabin, and S. Mirzadeh, (2000). "Evaluation of ${ }^{225}$ Ac for Vascular Targeted Radioimmunotherapy of Lung Tumors", Cancer Biotherapy and Radiopharmaceuticals, 15, 235.

Kikuchi, K., K. Kobayashi, K. Sueki, S. Suzuki, H. Nakahara, and Y. Achiba (1994). "Encapsulation of Radioactive ${ }^{159} \mathrm{Gd}$ and ${ }^{161} \mathrm{~Tb}$ Atoms in Fullerene Cages," J. Am. Chem. Soc. 116, 9775.

Knapp, Jr., F. F., (2001). "Future Prospects for Medical Radionuclide Production in The High Flux Isotope Reactor (HFIR) at The Oak Ridge National Laboratory (ORNL), " Ann. Nucl. Med. Sci. 14, 109-118.

Ksari-Habiles, Y. et al., (1997). "Superexchange and Magnetic Relaxation in Eu-Doped C 60 Phases," $J$. Phys. Chem. Solids 58, 1771-8.

Lewis J.S. et al., (2001). "Toxicity and Dosimetry of ${ }^{177}$ Lu-DOTA-Y3-Octreotate in a Rat Model," Int. J. Cancer 94, 873-7.

Lieser, K.H. (2001). Nuclear and Radiochemistry, Wiley-VCH, 185-6. 
Liu, S. and D.S. Edwards (2001). "Bifunctional Chelators for Therapeutic Lanthanide Radiopharmaceuticals," Bioconjugate Chem. 12, 7-34.

McDevitt, M.R. et al., (1998). "Radioimmunotherapy with Alpha-Emitting Nuclides," Eur. J. Nucl. Med. 25, 1341-1351.

McDevitt, M.R. et al (2001). "Tumor Therapy With Targeted Atomic Nanogenerators," Science 294, 1537.

Miederer, M., et al. (2003). "Pharmacokinetics, Dosimetry, and Toxicity of the Targetable Atomic Generator, ${ }^{225}$ Ac-HuM195, in Nonhuman Primates," J. Nuc. Med. 45, 129-137.

Milenic, D.E. E.D. Brasy, and M.W. Brechbiel (2004). “Antibody-Targeted Radioation Cancer Therapy," Nature Rev. Drug Disc. 3, 488-498.

Mirzadeh, S., (1998). "Generator-produced Alpha-Emitters", Appl. Radiat. Isot. 49(4), 345-349.

Mirzadeh, S., K.Kumar, and O.A. Gansow (1993). "The Chemical Fate of ${ }^{212}$ Bi-DOTA Formed by $\beta^{-}$ Decay of ${ }^{212} \mathrm{~Pb}(\mathrm{DOTA})^{2-}$," Radiochim. Acta 60, 1.

Mughabghab, S. F., M. Divadeenam and N. E. Holden, (1981). Neutron Cross Sections from Neutron Resonance Parameters and Thermal Cross Sections, Academic Press.

Mulford, D.A., D.A. Schienberg, and J.G. Jurcic (2004). "The Promise of Targeted $\alpha$-Particle Therapy," J. Nuc. Med. 46, 199S-204S.

Ohtsuki, T., et al., (1996). "Insertion of Be atoms in $\mathrm{C}_{60}$ Fullerene Cages: Be@, $\mathrm{C}_{60}$," Phys. Rev. Lett. 77, $3522-4$.

Ohtsuki, T., K. Ohno, K. Shiga, Y. Kawazoe, Y. Maruyama, and K. Masumoto (2000). "Systematic study of foreign-atom doped fullerenes by using a nuclear recoil method and their MD simulation," $J$. Chem. Phys. 112, 2834-2842.

Ohtsuki, T. et al., (2001). "Formation of Sb- and Te-Doped Fullerenes by Using Nuclear Recoil and Moelcular Dynamics Simulations," Phys. Rev. B 64, 125402.

Ohtsuki, T., K. Ohno, K. Shiga, Y. Kawazoe, and H. Yuki (2001). "Se atom Incorporation in Fullerenes by Using Nuclear Recoil and ab initio molecular dynamics calculations," Phys. Rev. B 65, 073402.

Ozdas, E., et al. (1995). "Superconductivity and Cation-Vacancy Ordering in the Rare-Earth Fulleride $\mathrm{Yb}_{2.75} \mathrm{C}_{60}$," Nature 375, 126-129.

Rausch, H. and T. Braun (2001). "Sublimation Behavior of $\mathrm{C}_{60}$ and of the Endohedral Radiofullerenes Formed by Nuclear Recoil Implosion via Neutron Irradiation," Chem. Phys. Lett. 350, 15-18.

Riva, P., (1999). Cancer Radioimmunotherapy: Present and Future, Harwood Academic, Amsterdam, The Netherlands.

Ruble, G. et al., (1996). "The Use of ${ }^{212} \mathrm{~Pb}-$ labeled Monoclonal Natibody in the Treatment of Murine Erythroleukemia," Int. J. Rad. Oncology Biol. Phys. 34, 609.

Saunders, M., H.A. Jimenez-Vasquez, R.J. Cross, S. Mroczkowski, M.L. Gross, D.E. Giblin, and R.J. Poreda (1994). "Incorporation of Helium Neon, Argon, Krypton, and Zenon Into Fullerenes Using High Pressure," J. Am. Chem. Soc. 116, 2193.

Schlom, J., et al. (1991). "Monoclonal Antibody-based Therapy of a Human Tumor Xenograft with a ${ }^{177}$ Lutetium-labeled Immunoconjugate," Cancer Res. 51, 2889-2896.

Schuster D.I., S.R. Wilson, A. N. Kirschner, R.F. Schinazi, S. Schluter-Wirtz, T. Barnett, S. Martin, J. Emerolieff, J. Tang, M. Brettrech, and A. Hirsch (2001). "Evaluation of the anti-HIV Potency of a Water Soluble Dendrimeric Fullerene Derivative", in preparation.

Sharkey, R.M., and D.M. Goldenberg (2005). "Perspectives on Cancer Therapy with Radiolabeled Monoclonal Antibodies," J. Nuc. Med. 46, 115S-127S.

Stein et al., (2001). "Radioimmunotherapy of a Human Lung Cancer Xenograft with Monoclonal Antibody RS7: Evaluation of 177Lu and Comparison of Its Efficacy with That of $90 \mathrm{Y}$ and Residualizing 131I," J. Nucl. Med. 42, 967-974.

Sueki, K., K. Akiyama, K. Kikuchi, and H. Nakahara (1998). "Specificity ofCarbon Cages Towards Oxidation States of Metal Atoms in Metallofullerenes," Chem. Phys. Lett. 291, 37.

Sueki, K., K. Akiyama, K. Kikuchi, and H. Nakahara (1999). "A C ${ }_{82}$ Carbon Cage Stable Toward Two Different Oxidation States of Endohedral Metal Atoms," J. Phys. Chem. 101, 1390. 
Thrash, T. P., D. W. Cagle, J. M. Alford, K. Wright, G. J. Ehrhardt, S. Mirzadeh, and L. J. Wilson, (1999). "Toward Fullerene-based Radiopharmaceuticals: High-Yield Neutron Activation of Endohedral ${ }^{165} \mathrm{Ho}$ Metallofullerenes," Chem. Phys. Lett. 308, 329.

Wilder, R.B., G.L, DeNardo, and S.J. DeNardo (1996). "Radioimmunotherapy:Recent Results and Future Directions", J. Clin. Oncology 14(4) 1383-1400.

Wilson, L. J., (1999). "Medical Applications of Fullerenes and Metallofullerenes," The Electrochemical Society Interface, (The Electrochemical Society, Inc., NJ.)

Wilson, S. R., and D. I. Schuster, (2000). "Biological Aspects of Fullerenes," in Fullerene Science and Technology, K. Kadish and R. Ruoff, eds., John Wiley and Sons, NY.

Zalutsky, M.R. and O.R. Pozzi (2004). "Radioimmunotherapy with $\alpha$-Particle Emitting Radionuclides," Q. J. Nuc. Med. Mol. Imag. 48, 289-296.

Zalutsky, M.R., X-G. Zhao, D. Bigner (2001). "High-Level Production of $\alpha$-Particle-Emitting ${ }^{211}$ At and Preparation of ${ }^{211}$ At-Labeled Antibodies for Clinical Use," J. Nuc. Med. 42, 1508-1515. 